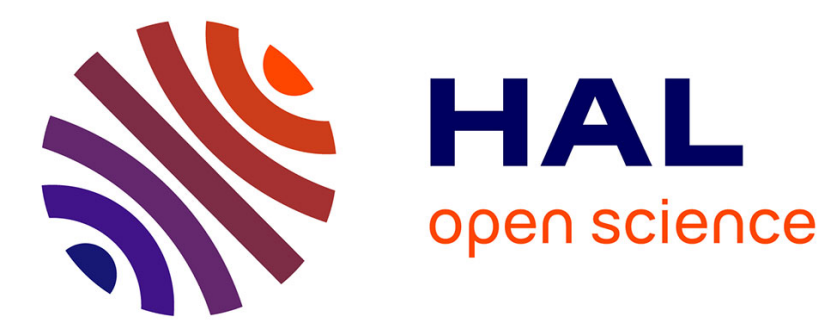

\title{
Wave propagation and non-local effects in periodic frame materials: Generalized continuum mechanics
}

Céline Chesnais, Claude Boutin, Stéphane Hans

\section{To cite this version:}

Céline Chesnais, Claude Boutin, Stéphane Hans. Wave propagation and non-local effects in periodic frame materials: Generalized continuum mechanics. Mathematics and Mechanics of Solids, 2015, 20 (8), pp 929-958. 10.1177/1081286513511092 . hal-00967960v2

\section{HAL Id: hal-00967960 https://hal.science/hal-00967960v2}

Submitted on 3 Dec 2015

HAL is a multi-disciplinary open access archive for the deposit and dissemination of scientific research documents, whether they are published or not. The documents may come from teaching and research institutions in France or abroad, or from public or private research centers.
L'archive ouverte pluridisciplinaire HAL, est destinée au dépôt et à la diffusion de documents scientifiques de niveau recherche, publiés ou non, émanant des établissements d'enseignement et de recherche français ou étrangers, des laboratoires publics ou privés. 


\section{Wave propagation and non-local effects in periodic frame materials: Generalized continuum mechanics}

Published online 29 November 2013 in Mathematics and Mechanics of Solids.

The original publication is available at $\mathrm{dx}$.doi.org/10.1177/1081286513511092

Céline Chesnais (corresponding author)

Université Paris-Est, IFSTTAR, GERS

14-20 boulevard Newton

Cité Descartes, Champs-sur-Marne

F-77447, Marne-la-Vallée Cedex 2, France

celine.chesnais@ifsttar.fr

Claude Boutin and Stéphane Hans

ENTPE/LGCB, CNRS-UMR 5513, CELYA, Université de Lyon

3 rue Maurice Audin

F-69518 Vaulx-en-Velin Cedex, France

claude.boutin@entpe.fr,stephane.hans@entpe.fr 


\begin{abstract}
Through the analysis of the wave propagation in infinite twodimensional periodic frame materials, this paper illustrates the complexity of their dynamic behavior. Assuming the frame size is small compared to the wavelength, the homogenization method of periodic discrete media coupled with normalization is used to identify the macroscopic behavior at the leading order. The method is applied on a frame material with the vertical elements stiffer than the horizontal elements. Such a material is highly anisotropic and presents a large contrast between the rigidities of the possible mechanisms. Thus the waves associated with different kinematics appear in different frequency ranges. Moreover, the stiffer elements can deform in bending at the macroscopic scale. The equivalent continuum is a second grade medium at the leading order and shear waves can be dispersive. A criterion is proposed to easily determine when this bending effect has to be taken into account.
\end{abstract}

\title{
1 Introduction
}

Periodic reticulated (or cellular) materials are obtained by repeating a unit cell made up of interconnected beams (or plates). Examples of such materials include wood, bone, foam, honeycomb but also stiffened plates and trussed beams used in aerospace and marine structures.

As the modeling of each cell element is computationally expensive, discrete reticulated materials are often replaced by an equivalent continuum. For frame materials with rigid joints (figure 1), which are the subject of this paper, a Cosserat or micropolar continuum is frequently used $[1,2,3]$. In this theory, rotations are introduced as independent degrees of freedom of the material particles in addition to the usual translational degrees of freedom $[4,5]$. It is a particular case of micromorphic continua, in which the particles can also experience deformation $[6,7,8]$. All these generalized continuum theories with additional degrees of freedom correspond to the class of the higher-order the- 


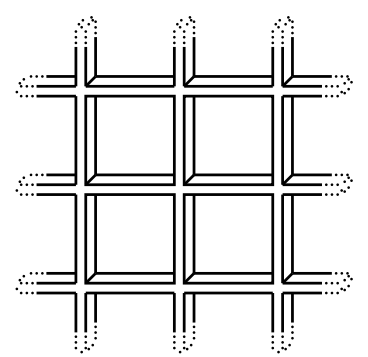

(a) SP material: the cell elements have similar properties

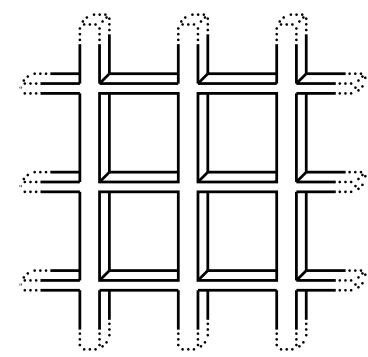

(b) SC material: vertical beams stiffer than horizontal beams

Figure 1: Examples of frame materials

ories. Another class of generalized continuum theories is constituted by the higher-grade theories, which incorporate the gradients of the strain in the strain energy density function $[9,10]$. It has been shown in [11] that trusses of articulated elastic beams can behave as such generalized continua. For a more detailed presentation of the different types of generalized continuum theories, the reader in referred to $[12,13]$.

The use of generalized continua to describe reticulated materials is motivated by the need to introduce a length scale to represent size effects. Indeed, when the macroscopic length $L$, defined by the specimen size or the loading wavelength, is on the same order as the cell size $\ell_{c}$, the mechanical properties depend on $L$ (see [14] and the references therein). On the contrary, when the condition of scale separation is respected $\left(L>>\ell_{c}\right)$, several studies based on homogenization methods $[15,16,17]$ have shown that frame materials behave as "classical" continua in the sense that only the translational degrees of freedom appear at the macroscopic scale and the strain energy density function does not depend on the gradients of the strain. These results were established for frame materials in which the cell elements have similar properties [figure 1(a)]. Such a material is called SP material afterwards.

In this work, we investigate the consequences on the wave propagation of 
the introduction of a stiffness contrast between the elements in the two directions [figure 1(b)]. This type of material is called SC material thereafter. In particular, it can behave as a generalized continuum, of the class of the highergrade theories, for wavelengths much greater than the cell size. Indeed, frame materials with a high stiffness contrast can be viewed as linear fiber reinforced composites. These composites are known for the existence of bending effects $[18,19,20]$, which have also been observed experimentally $[21,22]$. In SC materials, the combination of the bending effects with the other properties of frame materials described in [17] for SP materials (anisotropy, shear deformability much greater than compression deformability, local resonance) leads to an enriched local kinematics. Consequently, various types of waves generated by different mechanisms at different frequencies can travel in SC materials. For example, macroscopic shear waves can be dispersive because of the bending effects.

Since the study deals with situations with wavelengths much greater than the cell size, the macroscopic behavior is obtained by the homogenization method of periodic discrete media $[23,24]$. The stiffness contrast is introduced thanks to the scaling of the parameters during the normalization. This method enables an equivalent continuum to be rigorously derived from the knowledge of the properties of the unit cell. It is completely analytic, which provides a clear understanding of the role of each parameter. Moreover, it is always possible to come back to the unit cell and study its deformation. This constitutes a framework for the design of new materials with a prescribed behavior. This approach has proved to be a powerful tool for the study of reticulated materials and has been applied in various situations: see [25] for buckling analysis, [26] for the modeling of the cardiac tissue, [27] for the modeling of graphene sheets and $[28,29]$ for the dynamics of reticulated beams. The method also applies to situations with local resonance of the elements in bending [17]. Generally speaking, the interest of this approach is to give a "microscopic" basis to models 
which are otherwise postulated on purely logical grounds (e.g. [30] or [31, 32]).

The homogenization method is based on formal asymptotic expansions [33, 34 and the study focuses on the leading order. However, because of the lack of bracing of the frames $[35,36]$ and of the stiffness contrast between the elements, all the mechanisms have rigidities of different orders. Therefore, it is necessary to consider the higher order equations to obtain a complete description of the equivalent continuum. This leads to a singular perturbation problem [37]. Such problems are also encountered in plate and shell theories because of the contrast between the membrane behavior and pure bending [38]. But for SC frame materials, the kinematics is richer and the problem is more complex. The study of the convergence of the solution is out of the scope of this paper.

The latter is organized as follows. The framework of the study is presented in Section 2 and the implementation of the homogenization method of periodic discrete media (called HPDM afterwards) is described in Appendix A. Then, the complexity of the behavior of these materials is illustrated through the study of a particular case with a high stiffness contrast. This provides a list of the possible mechanisms and the associated kinematics. Section 3 presents the homogenized equations obtained in the absence of local resonance and the wave propagation in different frequency ranges is analyzed in Sections 4 to 7 . Section 8 deals with the behavior of real frame materials. After a summary of the main results and a comparison with [17] devoted to SP materials, a criterion is proposed to determine when the bending effects have to be taken into account without performing homogenization. Finally, in Section 9, a generic model which can degenerate into any of the previous macroscopic descriptions is built. 


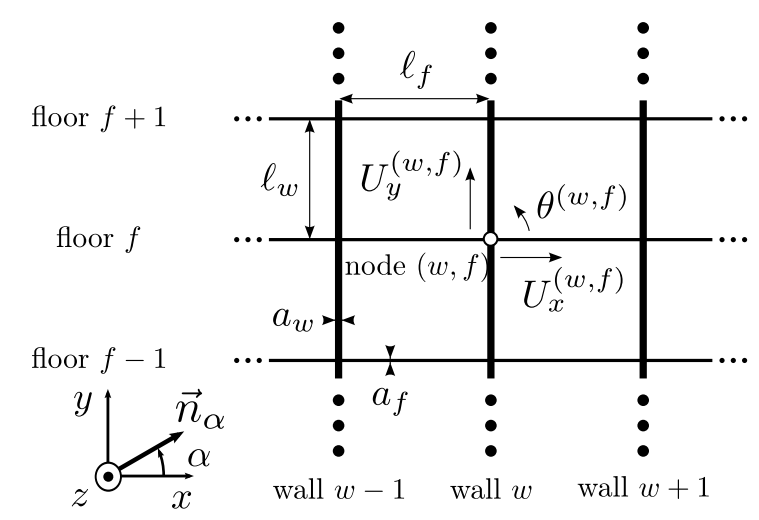

Figure 2: Notation for the frame material

\section{Framework of the study}

\section{$2.1 \quad$ Studied materials}

The studied materials are infinite and periodic in the plane $(x, y)$ of the wave propagation. They are made up of horizontal elements (called floors) supported by vertical elements (called walls). Elements are beams or plates behaving as Euler-Bernoulli beams in the plane $(x, y)$. They are linked by perfectly stiff and massless nodes. Thus, there is always a right angle between two elements. In this paper, we assume that the walls are stiffer than the floors.

The following notation will be used (figure 2):

- The characteristics of walls $(i=w)$ and floors $(i=f)$ are: $\ell_{i}$ length, $a_{i}$ thickness, $h_{i}$ depth according to the axis $z, A_{i}=a_{i} h_{i}$ cross-section area, $I_{i}=\left(h_{i} a_{i}^{3}\right) / 12$ second moment of area with respect to the axis $z, \rho_{i}$ density, $\bar{\rho}_{i}=\rho_{i} A_{i}$ mass per unit length and $E_{i}$ elastic modulus.

- The position of the node located at the intersection of the floor $f$ and the wall $w$ is determined either in a discrete manner by the ordered pair of integers $(w, f)$ (this is the local representation) or in a continuous manner by the coordinates $x=w \ell_{f}$ and $y=f \ell_{w}$ (this is the global representation). 


\section{$2.2 \quad$ Kinematic descriptors}

The study is conducted within the framework of the small strain theory and the linear elasticity. Moreover, the frame material vibrates at a given circular frequency $\omega$. As a result, every variable can be written in the following way: $X(x, y, t)=\Re\left(X(x, y) e^{i \omega t}\right)$ where $t$ is the time. Because of the linearity of the problem, the time dependence can be simplified and will be systematically omitted.

As explained in Appendix A, the HPDM method begins with the discretization of the dynamic balance. The study of the momentum balance of the whole frame material is exactly replaced by the study of the momentum balance of the nodes. Since the process is performed without loss of information, it is possible to use the motions of the nodes as kinematic descriptors of the motions of the cells. For the node $(w, f)$, the motion in the plane $(x, y)$ is described by the displacements in the two directions $U_{x}^{(w, f)}, U_{y}^{(w, f)}$ and by the node rotation $\theta^{(w, f)}$ (figure 2).

Then, the discrete description is transformed into a continuous description thanks to the principles of homogenization. The kinematic descriptors are considered as the discrete values of continuous functions of the space variables $x$ and $y$, which are expanded in powers of the scale ratio $\epsilon=\ell_{c} / L$ :

$$
\begin{aligned}
X^{(w, f)} & =X\left(\epsilon, x=w \ell_{f}, y=f \ell_{w}\right) \\
\text { with } X(\epsilon, x, y) & =X^{0}(x, y)+\epsilon X^{1}(x, y)+\epsilon^{2} X^{2}(x, y)+\ldots
\end{aligned}
$$

where $X$ stands for $U_{x}, U_{y}$ or $\theta$ and $X^{j}$ are continuous functions of order $j$. Afterwards, the physically observable motions of a given order in $\epsilon$ will be written with a tilde: $\tilde{X}^{j}(x, y)=\epsilon^{j} X^{j}(x, y)$. The exact motion of the node is $X^{(w, f)}$ whereas the function $X^{0}(x, y)$ is the approximation at the leading order which appears in the macroscopic description. In the sequel, we will only study the leading order of the descriptors unless it is explicitly specified.

Note that the macroscopic kinematic descriptors derived by homogenization 
have the same nature in discrete and continuous media: in both cases, the descriptor is the leading order of the motion of the cell. As a matter of fact, in usual weakly contrasted continuous composites (i.e. with no local resonance), the motion at the leading order is uniform in the cell and the average of each higher order is zero. Thus, the descriptor is equal not only to the mean motion of the whole cell, but also to the leading order motion of any point in the cell. For highly contrasted continuous composites (i.e. with local resonance), the kinematic descriptor of the homogenized behavior is the uniform displacement of the non-resonant part of the cell (see for instance $[39,40]$ ). Hence, for discrete media (with or without local resonance), the leading order motion of the node has the same physical meaning as the kinematic descriptor in continuous media.

\subsection{Normalization}

The stiffness contrast between the elements is introduced with the normalization. For real reticulated media, all the elements are frequently made of the same material. We therefore assume that the materials of the walls and the floors have similar properties and we only modify the thickness of the elements:

$$
\begin{array}{cl}
\frac{E_{f}}{E_{w}}=O(1), \quad \frac{\rho_{f}}{\rho_{w}}=O(1), \quad \frac{\ell_{f}}{\ell_{w}}=O(1), \quad \frac{h_{f}}{h_{w}}=O(1) \\
\frac{a_{w}}{\ell_{w}}=O\left(\epsilon^{1 / 2}\right), \quad \frac{a_{f}}{\ell_{w}}=O\left(\epsilon^{3 / 2}\right)
\end{array}
$$

The choice of fractions for the orders of magnitude of the thickness to length ratios is motivated by two reasons. First, the most interesting behaviors are observed for ratios close to $O(\epsilon)$. In this case, the local resonance of the elements in bending occurs in the same frequency range as the propagation of macroscopic compressional waves [17]. The second reason is that calculations are simpler when the order of magnitude of the ratio $a_{f} / a_{w}$ is an integer. This generally makes it possible to only consider the whole powers of $\epsilon$ in the asymptotic 
expansions [as in Eq. (1)]. However, we will see in Section 7 that, due to the local resonance in bending, the order of magnitude of the effective mass is a multiple of $1 / 4$. In this case, the descriptors have to be expanded according to the powers of $\epsilon^{1 / 4}$ for all the inertial terms to be balanced by an elastic term:

$$
\begin{aligned}
X(\epsilon, x, y)=X^{0}(x, y) & +\epsilon^{1 / 4} X^{1 / 4}(x, y)+\epsilon^{1 / 2} X^{1 / 2}(x, y) \\
+ & \epsilon^{3 / 4} X^{3 / 4}(x, y)+\epsilon X^{1}(x, y)+\epsilon^{5 / 4} X^{5 / 4}(x, y)+\ldots
\end{aligned}
$$

For the scaling of the circular frequency, the reference $\omega_{r}$ is taken by convention as:

$$
\omega_{r}=\frac{1}{L} \sqrt{\frac{E_{w}}{\rho_{w}}}
$$

Waves can deform the elements in two different ways: in bending and in tensioncompression. Since the first mechanism is more flexible and there is a stiffness contrast between the walls and the floors, the various types of waves appear in four frequency ranges:

$$
\frac{\omega}{\omega_{r}}=O\left(\epsilon^{2}\right), \quad \frac{\omega}{\omega_{r}}=O\left(\epsilon^{3 / 2}\right), \quad \frac{\omega}{\omega_{r}}=O\left(\epsilon^{1 / 2}\right), \quad \frac{\omega}{\omega_{r}}=O(1)
$$

\section{Homogenized equations}

This section and the next present the behavior of the frame material at the lowest circular frequencies giving a dynamic description at the leading order: $\omega=O\left(\epsilon^{2} \omega_{r}\right)$. In that case, the implementation of the HPDM method provides the following equations corresponding to the balance of momentum in the $x$ direction for orders 0 to 3 , the balance of momentum in the $y$ direction for 
orders 0 to 4 and the balance of moment of momentum for orders 0 to 3 .

$$
\begin{aligned}
& \left\{\begin{array}{l}
E_{x} \frac{\partial^{2} \tilde{U}_{x}^{0}}{\partial x^{2}}+G_{w}\left(\frac{\partial \tilde{\theta}^{0}}{\partial y}+\frac{\partial^{2} \tilde{U}_{x}^{0}}{\partial y^{2}}\right)=0 \\
E_{x} \frac{\partial^{2} \tilde{U}_{x}^{1}}{\partial x^{2}}+G_{w}\left(\frac{\partial \tilde{\theta}^{1}}{\partial y}+\frac{\partial^{2} \tilde{U}_{x}^{1}}{\partial y^{2}}\right)=0 \\
E_{x} \frac{\partial^{2} \tilde{U}_{x}^{2}}{\partial x^{2}}+G_{w}\left(\frac{\partial \tilde{\theta}^{2}}{\partial y}+\frac{\partial^{2} \tilde{U}_{x}^{2}}{\partial y^{2}}\right)-E I_{z} \frac{\partial^{4} \tilde{U}_{x}^{0}}{\partial y^{4}}=0 \\
E_{x} \frac{\partial^{2} \tilde{U}_{x}^{3}}{\partial x^{2}}+G_{w}\left(\frac{\partial \tilde{\theta}^{3}}{\partial y}+\frac{\partial^{2} \tilde{U}_{x}^{3}}{\partial y^{2}}\right)-E I_{z} \frac{\partial^{4} \tilde{U}_{x}^{1}}{\partial y^{4}}+M_{w} \omega^{2} \tilde{U}_{x}^{0}=0
\end{array}\right. \\
& \left\{\begin{array}{l}
\forall j \in\{0,1,2,3\} \quad E_{y} \frac{\partial^{2} \tilde{U}_{y}^{j}}{\partial y^{2}}=0 \\
E_{y} \frac{\partial^{2} \tilde{U}_{y}^{4}}{\partial y^{2}}+G_{f}\left(-\frac{\partial \tilde{\theta}^{0}}{\partial x}+\frac{\partial^{2} \tilde{U}_{y}^{0}}{\partial x^{2}}\right)+M_{w} \omega^{2} \tilde{U}_{y}^{0}=0
\end{array}\right. \\
& \left\{\begin{array}{l}
\forall j \in\{0,1,2\} \quad G_{w}\left(\tilde{\theta}^{j}+\frac{\partial \tilde{U}_{x}^{j}}{\partial y}\right)=0 \\
G_{w}\left(\tilde{\theta}^{3}+\frac{\partial \tilde{U}_{x}^{3}}{\partial y}\right)-G_{f}\left(-\tilde{\theta}^{0}+\frac{\partial \tilde{U}_{y}^{0}}{\partial x}\right)=0
\end{array}\right.
\end{aligned}
$$

with:

$M_{w}=\bar{\rho}_{w} / \ell_{f} \quad:$ contribution of the walls to the mass per unit surface $\left(\mathrm{kg} / \mathrm{m}^{2}\right)$ $M_{f}=\bar{\rho}_{f} / \ell_{w} \quad:$ contribution of the floors to the mass per unit surface $\left(\mathrm{kg} / \mathrm{m}^{2}\right)$ (it will appear in other sections)

$E_{x}=E_{f} A_{f} / \ell_{w}:$ elastic modulus in the $x$ direction $(\mathrm{N} / \mathrm{m})$ $E_{y}=E_{w} A_{w} / \ell_{f}:$ elastic modulus in the $y$ direction $(\mathrm{N} / \mathrm{m})$ $G_{w}=12 \frac{E_{w} I_{w}}{\ell_{w}^{2} \ell_{f}}:$ modulus of the local bending of the walls $(\mathrm{N} / \mathrm{m})$ $G_{f}=12 \frac{E_{f} I_{f}}{\ell_{w} \ell_{f}^{2}} \quad:$ modulus of the local bending of the floors $(\mathrm{N} / \mathrm{m})$ $E I_{z}=E_{w} I_{w} / \ell_{f}:$ modulus of the macroscopic bending (N.m)

The macroscopic parameters are integrated over the depth of the frame material and so they do not have the usual units. Their expressions only depend on the elastostatic properties of the cell elements because of the quasi-static state 
at the local scale. Since beams are more flexible in bending than in tensioncompression and the floors are thinner than the walls, the various parameters have different orders of magnitude:

$$
\begin{gathered}
\frac{M_{f}}{M_{w}}=O(\epsilon), \quad \frac{G_{f}}{G_{w}}=O\left(\epsilon^{3}\right), \\
\frac{E_{x}}{E_{y}}=O(\epsilon), \quad \frac{G_{f}}{E_{y}}=O\left(\epsilon^{4}\right), \quad \frac{E I_{z}}{E_{y} L^{2}}=O\left(\epsilon^{3}\right)
\end{gathered}
$$

In this work, we assume that all the possible motions have the same importance. As a result, the corresponding terms appear in equations of different orders. This is the reason why we have to consider higher order equations to obtain a complete description of the macroscopic behavior of the material.

The balance equations (4) contain inertial terms [in Eqs. $(x 3)$ and $(y 4)$ ] and five types of elastic terms:

$$
\begin{array}{rlrl}
\tilde{\sigma}_{x x}^{j+1} & =E_{x} \frac{\partial \tilde{U}_{x}^{j}}{\partial x} & \tilde{\tau}_{x y}^{j+1}=G_{w}\left(\tilde{\theta}^{j}+\frac{\partial \tilde{U}_{x}^{j}}{\partial y}\right) \\
\tilde{\sigma}_{y y}^{j}=E_{y} \frac{\partial \tilde{U}_{y}^{j}}{\partial y} & \tilde{\tau}_{y x}^{j+4}=G_{f}\left(-\tilde{\theta}^{j}+\frac{\partial \tilde{U}_{y}^{j}}{\partial x}\right) \\
\tilde{\mu}_{z}^{j+3}=-E I_{z} \frac{\partial^{2} \tilde{U}_{x}^{j}}{\partial y^{2}} & &
\end{array}
$$

With this notation, all the terms with the same superscript have the same order of magnitude.

The balance of moment of momentum shows that the first orders of $\tau_{x y}$ vanish and that the first non-zero terms of $\tau_{x y}$ and $\tau_{y x}$, i.e. $\tilde{\tau}_{x y}^{4}$ generated by the displacement of order 3 and $\tilde{\tau}_{y x}^{4}$ generated by the displacement of order 0 , are equal [Eq. (m3) of (4)]. Moreover, it is possible to eliminate the node rotation $\tilde{\theta}^{0}$ thanks to Eq. ( $\left.m 0\right)$ of (4):

$$
\tilde{\tau}_{x y}^{4}=\tilde{\tau}_{y x}^{4}=\tilde{\tau}^{4}=G_{f}\left(\frac{\partial \tilde{U}_{y}^{0}}{\partial x}+\frac{\partial \tilde{U}_{x}^{0}}{\partial y}\right)
$$

This means that the node rotation is a "hidden" degree of freedom. The calculation of $\tilde{\theta}^{0}$ is only required for the study of the deformation of the unit cell at the local scale. The behavior of the frame material at the macroscopic scale is 


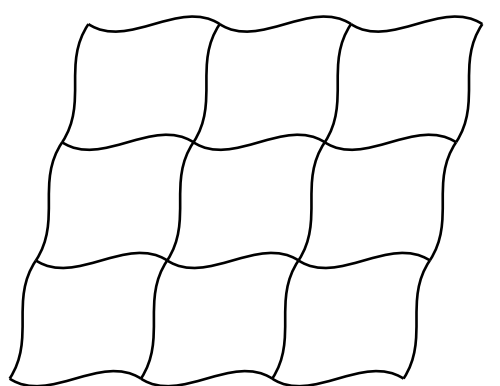

(a) Local bending of the elements

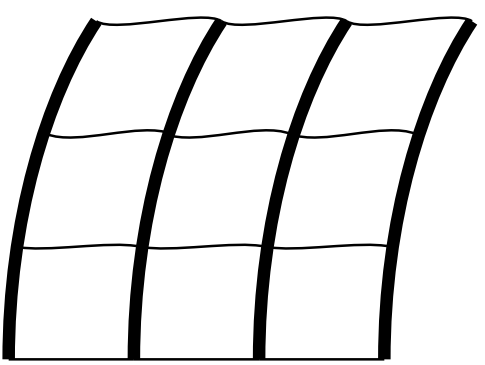

(b) Bending of the walls at the macroscopic scale and local bending of the floors

Figure 3: Comparison of the two types of bending

completely described by the translational degrees of freedom and the balance of momentum in the $x$ and $y$ directions.

Finally, these equations contain four types of elastic terms $\left(\sigma_{x x}, \sigma_{y y}, \tau\right.$ and $\mu_{z}$ ) corresponding to the four possible mechanisms and to four different kinematics. The terms $\sigma_{x x}$ and $\sigma_{y y}$ are related to the tension-compression of the floors and the walls, respectively. The term $\tau$ is associated with the shear strain of the unit cell. Such a deformation is due to the local bending of the elements [figure 3(a)]. But, because of the stiffness contrast between the walls and the floors, only the floors are deformed in SC materials and the shear modulus is degenerate: $G \approx G_{f}$. This is consistent with the connection in series of $G_{w}$ and $G_{f}$ evidenced in [17]. In this previous work devoted to SP materials, the shear modulus was given by:

$$
\frac{1}{G}=\frac{1}{G_{f}}+\frac{1}{G_{w}}
$$

Local bending corresponds to the deformation of the elements between two neighboring nodes. It must not be confused with the last mechanism described by $\mu_{z}$. The second derivative of $\tilde{U}_{x}^{0}$ according to a continuous space variable indicates that the walls experience bending at the macroscopic scale [figure 3(b)]. 


\section{Local bending of the floors}

We now examine the properties of the waves propagating in SC materials. The study focuses on plane waves and the displacement field is sought in the following way (remember that the time dependence $\exp (i \omega t)$ is omitted):

$$
\vec{U}(\epsilon, \vec{x})=\vec{u}^{0} \exp \left[-i k(\alpha) \vec{n}_{\alpha} \cdot \vec{x}\right]+\epsilon \vec{U}^{1}(\vec{x})+\epsilon^{2} \vec{U}^{2}(\vec{x})+\ldots
$$

Only the expression of the displacement field at the leading order

$\vec{U}^{0}(\vec{x})=\vec{u}^{0} \exp \left[-i k(\alpha) \vec{n}_{\alpha} \cdot \vec{x}\right]$ is imposed. It corresponds to a plane wave with an amplitude $\vec{u}^{0}$ and a wave number $k(\alpha)$ traveling in the direction $\vec{n}_{\alpha}$ (figure 2). The correctors could be determined without any additional assumptions by the resolution of the higher order equations as in [41]. Their study is out of the scope of this paper but the following property will be used. As the material is infinite and the macroscopic field $\vec{U}^{0}$ is invariant under a translation perpendicular to the direction of propagation, the correctors must also be invariant under such a translation.

We begin with the lowest frequencies: $\omega=O\left(\epsilon^{2} \omega_{r}\right)$. The balance equations are the same as Eqs. (4) of Section 3. The equations describing the balance of moment of momentum are used to eliminate the node rotation $\tilde{\theta}^{0}$. Then the 
expression (8) of the displacement field is introduced:

$$
\begin{aligned}
& \left\{\begin{array}{l}
-E_{x} k^{2}(\alpha) \cos ^{2}(\alpha) \tilde{u}_{x}^{0} \exp \left[-i k(\alpha) \vec{n}_{\alpha} \cdot \vec{x}\right]=0 \\
E_{x} \frac{\partial^{2} \tilde{U}_{x}^{1}}{\partial x^{2}}=0 \\
E_{x} \frac{\partial^{2} \tilde{U}_{x}^{2}}{\partial x^{2}}-E I_{z} k^{4}(\alpha) \sin ^{4}(\alpha) \tilde{u}_{x}^{0} \exp \left[-i k(\alpha) \vec{n}_{\alpha} \cdot \vec{x}\right]=0
\end{array}\right. \\
& \left\{\begin{array}{l}
-E_{y} k^{2}(\alpha) \sin ^{2}(\alpha) \tilde{u}_{y}^{0} \exp \left[-i k(\alpha) \vec{n}_{\alpha} \cdot \vec{x}\right]=0 \\
\forall j \in\{1,2,3\} \quad E_{y} \frac{\partial^{2} \tilde{U}_{y}^{j}}{\partial y^{2}}=0 \\
E_{y} \frac{\partial^{2} \tilde{U}_{y}^{4}}{\partial y^{2}}+\left(-G_{f} k^{2}(\alpha) \cos (\alpha)\left(\sin (\alpha) \tilde{u}_{x}^{0}+\cos (\alpha) \tilde{u}_{y}^{0}\right)\right. \\
\left.\quad+M_{w} \omega^{2} \tilde{u}_{y}^{0}\right) \exp \left[-i k(\alpha) \vec{n}_{\alpha} \cdot \vec{x}\right]=0
\end{array}\right.
\end{aligned}
$$

Equations $(x 0)$ and $(y 0)$ show that $\vec{u}^{0}=\overrightarrow{0}$ for $\cos (\alpha) \neq 0$ and $\sin (\alpha) \neq 0$. Thus waves cannot travel diagonally in this frequency range. For $\cos (\alpha)=0$ (propagation in the $y$ direction), Eq. ( $y 0)$ implies that $\tilde{u}_{y}^{0}=0$. Moreover, in Eq. $(x 2)$, the corrector must be invariant under a translation parallel to the $x$ direction: $\partial_{x}^{2} \tilde{U}_{x}^{2}=0$. So Eq. $(x 2)$ implies that $\tilde{u}_{x}^{0}=0$ and the propagation is also impossible in the $y$ direction.

As a result, waves can only travel in the $x$ direction $[\sin (\alpha)=0]$. We deduce from Eq. $(x 0)$ that $\tilde{u}_{x}^{0}=0$. This means that the waves are polarized in the $y$ direction and that they are pure shear waves. Now the problem is invariant under a translation parallel to the $y$ direction. In Eq. $(y 4)$, the term with the corrector vanishes and the other terms give the wave number:

$$
k(\alpha=0)=\omega \sqrt{\frac{M_{w}}{G_{f}}} \Rightarrow c(\alpha=0)=\sqrt{\frac{G_{f}}{M_{w}}}
$$

The speed $c(\alpha=0)$ has the same expression as the speed of shear waves in a classical elastic medium. But for SC materials, the mass and the shear modulus are degenerate. Since the thicknesses of the walls and the floors have different orders of magnitude, there is a large contrast between $M_{w}$ and $M_{f}$ and between 
$G_{w}$ and $G_{f}$ [Eq. (5)]. Only $M_{w}$ and $G_{f}$ appear at the leading order. The waves are generated by the local bending of the floors (figure 4) which is the most flexible mechanism. The propagation in the other directions is prevented by the stiff walls. At these low frequencies, they still have a quasi-static behavior at the macroscopic scale.

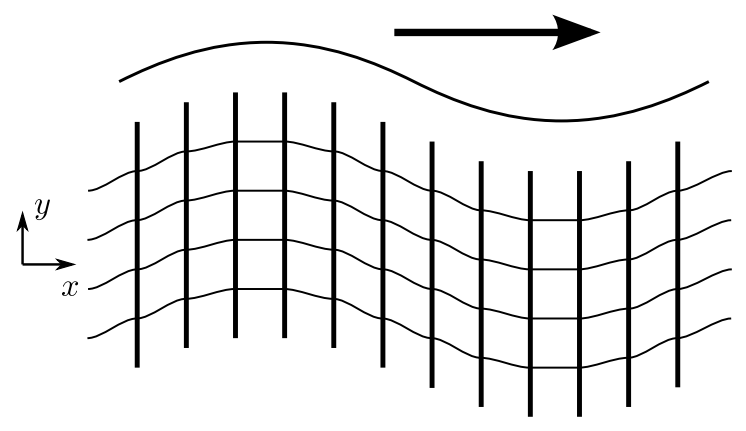

Figure 4: Shear wave generated by the local bending of the floors

\section{Bending waves in the walls}

The circular frequency $\omega$ is increased up to $O\left(\epsilon^{3 / 2} \omega_{r}\right)$ so that the inertia forces balance the term associated with the macroscopic bending of the walls. The HPDM method provides the following equations for the balance of momentum. The equations describing the balance of moment of momentum are the same as 
in Eqs. (4) of Section 3 and are used to eliminate $\tau_{x y}$.

$$
\begin{aligned}
& \begin{cases}E_{x} \frac{\partial^{2} \tilde{U}_{x}^{0}}{\partial x^{2}}=0 & (x 0) \\
E_{x} \frac{\partial^{2} \tilde{U}_{x}^{1}}{\partial x^{2}}=0 & (x 1) \\
E_{x} \frac{\partial^{2} \tilde{U}_{x}^{2}}{\partial x^{2}}-E I_{z} \frac{\partial^{4} \tilde{U}_{x}^{0}}{\partial y^{4}}+M_{w} \omega^{2} \tilde{U}_{x}^{0}=0 & (x 2)\end{cases} \\
& \left\{\begin{array}{l}
\forall j \in\{0,1,2\} \quad E_{y} \frac{\partial^{2} \tilde{U}_{y}^{j}}{\partial y^{2}}=0 \\
E_{y} \frac{\partial^{2} \tilde{U}_{y}^{3}}{\partial y^{2}}+M_{w} \omega^{2} \tilde{U}_{y}^{0}=0
\end{array}\right.
\end{aligned}
$$

The introduction of the expression (8) of the displacement field leads to the following results. As Eqs. $(x 0)$ and $(y 0)$ are identical to Eqs. $(x 0)$ and $(y 0)$ of (9) in Section 4, waves cannot travel diagonally. Moreover, Eqs. $(x 0)$ and (y3) show that the propagation is impossible in the $x$ direction.

For the propagation in the $y$ direction, we deduce from Eq. $(y 0)$ that the waves are polarized in the $x$ direction. As the problem is invariant under a translation parallel to the $x$ direction, the term $E_{x} \partial_{x}^{2} \tilde{U}_{x}^{2}$ in Eq. $(x 2)$ vanishes and the wave number is given by the other terms:

$$
\begin{gathered}
E_{x} \frac{\partial^{2} \tilde{U}_{x}^{2}}{\partial x^{2}}+\left[-E I_{z} k^{4}\left(\alpha=\frac{\pi}{2}\right)+M_{w} \omega^{2}\right] \tilde{u}_{x}^{0} \exp \left[-i k\left(\alpha=\frac{\pi}{2}\right) y\right]=0 \\
\Rightarrow \quad k\left(\alpha=\frac{\pi}{2}\right)=\sqrt[4]{\frac{\omega^{2} M_{w}}{E I_{z}}}
\end{gathered}
$$

Even if the motion $\tilde{U}_{x}^{0}(y)$ still corresponds to macroscopic pure shear waves, the expression of the wave number is completely different. This means that the waves are generated by another mechanism. They are due to the propagation in the walls of bending waves synchronized by the floors which are very stiff in tension-compression (figure 5). Consequently, the macroscopic shear waves have different properties. In particular, there is dispersion (figure 6) and the speed of the waves varies according to $\sqrt{\omega}$ :

$$
c\left(\alpha=\frac{\pi}{2}\right)=\sqrt[4]{\frac{\omega^{2} E I_{z}}{M_{w}}}
$$




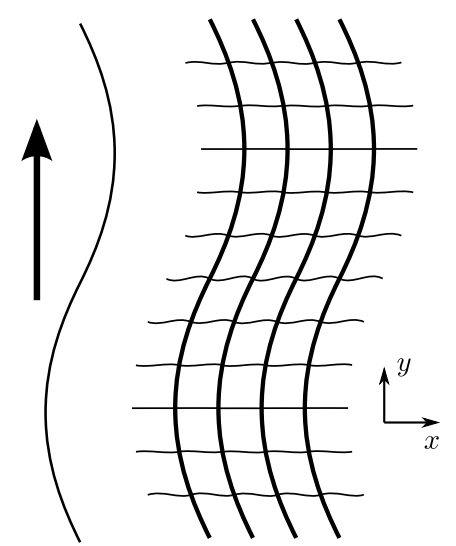

Figure 5: Shear wave generated by the propagation of bending waves in the walls

Macroscopic shear waves cannot travel diagonally because it requires to deform the elements in tension-compression. For Euler-Bernoulli beams, this mechanism is more rigid than bending and the frequency has to be increased.

\section{Tension-compression and bending resonance of the floors}

\subsection{Leading order and wave propagation}

We consider here the third frequency range at which wave propagation can occur: $\omega=O\left(\epsilon^{1 / 2} \omega_{r}\right)$. In that case, the floors are in resonance in bending, which means that the bending wavelength in the floors $\lambda_{b f}$ is of the same order as their length $\ell_{f}$. However, the compression wavelength in the floors $\lambda_{c f}$ remains much greater and homogenization still applies with the procedure presented in Appendix A (also see [17]). Moreover, the walls being stiffer, they have a quasistatic behavior at the local scale for both mechanisms. As a result, during the implementation of the HPDM method, we have to keep the complete expressions 


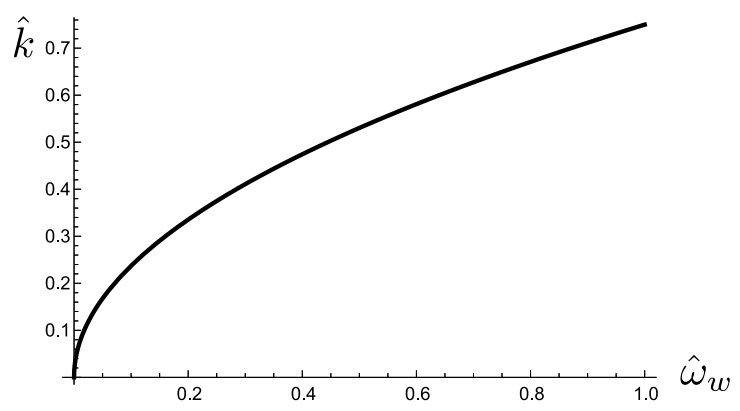

Figure 6: Variation of the dimensionless wave number $\hat{k}=k\left(\alpha=\frac{\pi}{2}\right) \times \frac{\ell_{w}}{2 \pi}$ according to the dimensionless frequency $\hat{\omega}_{w}$ for macroscopic shear waves in the $y$ direction. The dimensionless frequency is defined by $\hat{\omega}_{w}=\omega\left(\frac{2 \ell_{w}}{3 \pi}\right)^{2} \sqrt{\frac{\bar{\rho}_{w}}{E_{w} I_{w}}} \approx \frac{\omega}{\omega_{w 1}}$ where $\omega_{w 1}$ is the circular frequency of the first bending mode of the floors with two fixed ends.

of the shear force and the bending moment in the floors but all the other forces and moments can be expanded. Then, the following equations are obtained. Equation $(m 0)$ is used to eliminate the node rotation $\tilde{\theta}^{0}$ in the other equations.

$$
\begin{aligned}
& E_{x} \frac{\partial^{2} \tilde{U}_{x}^{0}}{\partial x^{2}}+M_{w} \omega^{2} \tilde{U}_{x}^{0}=0 \\
& \left\{\begin{array}{l}
E_{y} \frac{\partial^{2} \tilde{U}_{y}^{0}}{\partial y^{2}}=0 \\
E_{y} \frac{\partial^{2} \tilde{U}_{y}^{1}}{\partial y^{2}}+M_{w} \omega^{2} \tilde{U}_{y}^{0}=0 \\
G_{w}\left(\tilde{\theta}^{0}+\frac{\partial \tilde{U}_{x}^{0}}{\partial y}\right)=0
\end{array}\right.
\end{aligned}
$$

For the study of the wave propagation, the expression (8) of the displacement field is introduced in Eqs. $(x 0)$ and $(y 0)$.

$$
\begin{array}{r}
\left(-E_{x} k^{2}(\alpha) \cos ^{2}(\alpha)+M_{w} \omega^{2}\right) \tilde{u}_{x}^{0} \exp \left[-i k(\alpha) \vec{n}_{\alpha} \cdot \vec{x}\right]=0 \\
-E_{y} k^{2}(\alpha) \sin ^{2}(\alpha) \tilde{u}_{y}^{0} \exp \left[-i k(\alpha) \vec{n}_{\alpha} \cdot \vec{x}\right]=0
\end{array}
$$


The existence of a non-zero solution implies that:

$$
-E_{x} k^{2}(\alpha) \cos ^{2}(\alpha)+M_{w} \omega^{2}=0 \quad \text { or } \quad \sin (\alpha)=0
$$

Moreover, for:

$$
-E_{x} k^{2}(\alpha) \cos ^{2}(\alpha)+M_{w} \omega^{2} \neq 0 \quad \text { and } \quad \sin (\alpha)=0
$$

waves travel in the $x$ direction and Eq. $(x 0)$ of (14) imposes $\tilde{u}_{x}^{0}=0$. As the problem is invariant under a translation parallel to the $y$ direction, Eq. $(y 1)$ of (13) gives $\tilde{u}_{y}^{0}=0$ and the propagation is impossible. Therefore, the following conditions are always satisfied:

$$
-E_{x} k^{2}(\alpha) \cos ^{2}(\alpha)+M_{w} \omega^{2}=0 \quad \text { and } \quad \tilde{u}_{y}^{0}=0
$$

The expression of the wave number is:

$$
k(\alpha)=\frac{\omega}{|\cos (\alpha)|} \sqrt{\frac{M_{w}}{E_{x}}} \Rightarrow c(\alpha)=|\cos (\alpha)| \sqrt{\frac{E_{x}}{M_{w}}}
$$

Waves can travel in all the directions except in the $y$ direction $[\cos (\alpha)=0]$. But whatever the direction of propagation, the waves are polarized in the direction of the floors. Thus, the waves traveling in the $x$ direction are pure compressional waves whereas the waves traveling in the other directions are shear-compression waves. This type of polarization was called X-mode in [17]. The speed depends on the direction of propagation and is maximal for the waves traveling in the $x$ direction. However, it does not vary with the frequency.

These macroscopic waves are generated at the local scale by the propagation in the floors of compressional waves which can be out-of-phase as explained in figure 7. The gray grid represents the frames before deformation. All the nodal displacements are in the $x$ direction as shown by the arrows on the left. The motions are caused by the propagation of pure compressional waves in the floors. Their amplitude is represented by the gray sinusoids at the bottom of the figure and their wavelength is $\Lambda(\alpha=0)=(2 \pi / \omega) \sqrt{\left(E_{x} / M_{w}\right)}$. The waves in the floors are out-of-phase, so that all the points on a straight line perpendicular 


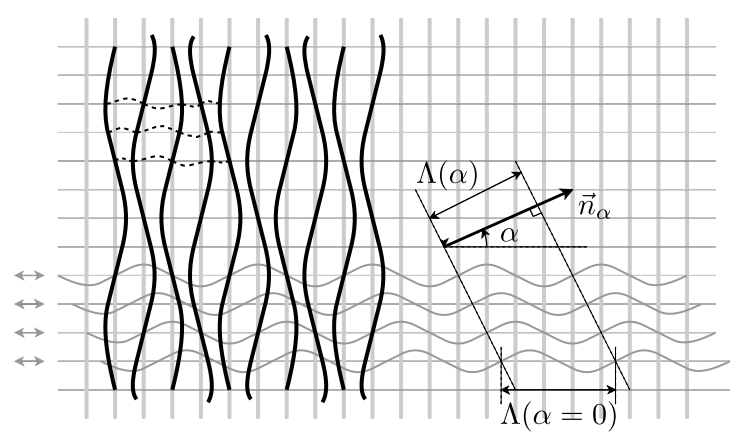

Figure 7: Macroscopic shear-compression wave traveling in the direction $\vec{n}_{\alpha}$ with the X-mode

to the direction $\vec{n}_{\alpha}$ have the same displacement. This is the reason why, at the macroscopic scale, we observe a wave traveling in the direction $\vec{n}_{\alpha}$. The wavelength of the macroscopic wave $\Lambda(\alpha)$ is the projection of $\Lambda(\alpha=0)$ onto the direction $\vec{n}_{\alpha}$. Consequently, the wavelength (and therefore the speed) is maximal when the macroscopic wave travels in the $x$ direction. Moreover, this mechanism cannot generate a macroscopic wave traveling in the $y$ direction.

Because of the phase difference between the compressional waves in the floors, the unit cells experience a shear strain. This induces the bending of the walls at the macroscopic scale represented by the vertical black sinusoids. Since the walls are not in resonance, it corresponds to their total deformation. Equation $(m 0)$ of (13) shows that the bending of the walls results in the rotation of the nodes. The floors are subjected to local bending in addition to the compressional waves. For the considered frequency range, the floors are in resonance and their deformation is drawn with the dotted lines in the top left corner of figure 7 .

\subsection{Higher orders and local resonance}

The previous section shows that the bending resonance of the floors has no effect on the wave propagation at the leading order. To identify the consequences of 
this phenomenon, we must therefore analyze the higher order equations. They are written below and the equations describing the balance of moment of momentum are used to eliminate the node rotation in the other equations.

$$
\begin{aligned}
& \left\{\begin{array}{l}
E_{x} \frac{\partial^{2} \tilde{U}_{x}^{0}}{\partial x^{2}}+M_{w} \omega^{2} \tilde{U}_{x}^{0}=0 \\
E_{x} \frac{\partial^{2} \tilde{U}_{x}^{1}}{\partial x^{2}}+M_{w} \omega^{2} \tilde{U}_{x}^{1}+M_{f} \omega^{2} \tilde{U}_{x}^{0}=0
\end{array}\right. \\
& \left\{\begin{array}{l}
E_{y} \frac{\partial^{2} \tilde{U}_{y}^{0}}{\partial y^{2}}=0 \\
E_{y} \frac{\partial^{2} \tilde{U}_{y}^{1}}{\partial y^{2}}+M_{w} \omega^{2} \tilde{U}_{y}^{0}=0 \\
E_{y} \frac{\partial^{2} \tilde{U}_{y}^{2}}{\partial y^{2}}+M_{w} \omega^{2} \tilde{U}_{y}^{1}+M_{f} f\left(\hat{\omega}_{f}\right) \omega^{2} \tilde{U}_{y}^{0}=0 \\
G_{w}\left(\tilde{\theta}^{0}+\frac{\partial \tilde{U}_{x}^{0}}{\partial y}\right)=0 \\
G_{w}\left(\tilde{\theta}^{1}+\frac{\partial \tilde{U}_{x}^{1}}{\partial y}\right)=0 \\
G_{w}\left(\tilde{\theta}^{2}+\frac{\partial \tilde{U}_{x}^{2}}{\partial y}\right)+\frac{\ell_{w}^{2}}{15} M_{w} \omega^{2} \frac{\partial \tilde{U}_{x}^{0}}{\partial y}=0 \\
G_{w}\left(\tilde{\theta}^{3}+\frac{\partial \tilde{U}_{x}^{3}}{\partial y}\right)+\frac{\ell_{w}^{2}}{15} M_{w} \omega^{2} \frac{\partial \tilde{U}_{x}^{1}}{\partial y}-\tau\left(\hat{\omega}_{f}\right)=0
\end{array}\right.
\end{aligned}
$$

with

$$
\begin{aligned}
\hat{\omega}_{f} & =\omega\left(\frac{2 \ell_{f}}{3 \pi}\right)^{2} \sqrt{\frac{\bar{\rho}_{f}}{E_{f} I_{f}}} \approx \frac{\omega}{\omega_{f 1}} \\
f(\hat{\omega}) & =\frac{8}{3 \pi \sqrt{\hat{\omega}} f_{o}(\hat{\omega})} \sin \left(\frac{3 \pi}{4} \sqrt{\hat{\omega}}\right) \sinh \left(\frac{3 \pi}{4} \sqrt{\hat{\omega}}\right) \\
f_{o}(\hat{\omega}) & =\sin \left(\frac{3 \pi}{4} \sqrt{\hat{\omega}}\right) \cosh \left(\frac{3 \pi}{4} \sqrt{\hat{\omega}}\right)+\sinh \left(\frac{3 \pi}{4} \sqrt{\hat{\omega}}\right) \cos \left(\frac{3 \pi}{4} \sqrt{\hat{\omega}}\right) \\
f_{e}(\hat{\omega}) & =\sin \left(\frac{3 \pi}{4} \sqrt{\hat{\omega}}\right) \cosh \left(\frac{3 \pi}{4} \sqrt{\hat{\omega}}\right)-\sinh \left(\frac{3 \pi}{4} \sqrt{\hat{\omega}}\right) \cos \left(\frac{3 \pi}{4} \sqrt{\hat{\omega}}\right)
\end{aligned}
$$




$$
\begin{aligned}
\tau\left(\hat{\omega}_{f}\right)=\frac{\pi G_{f} \sqrt{\hat{\omega}_{f}}}{8 f_{e}\left(\hat{\omega}_{f}\right)}\left(-4 \sin \left(\frac{3 \pi}{4} \sqrt{\hat{\omega}_{f}}\right) \sinh \left(\frac{3 \pi}{4} \sqrt{\hat{\omega}_{f}}\right) \tilde{\theta}^{0}\right. \\
\left.\quad+\frac{3 \pi \sqrt{\hat{\omega}_{f}}}{2 f_{o}\left(\hat{\omega}_{f}\right)}\left(\cosh \left(\frac{3 \pi}{2} \sqrt{\hat{\omega}_{f}}\right)-\cos \left(\frac{3 \pi}{2} \sqrt{\hat{\omega}_{f}}\right)\right) \frac{\partial \tilde{U}_{y}^{0}}{\partial x}\right)
\end{aligned}
$$

Because of the local resonance, the shear force and the bending moment in the floors are not expanded in the HPDM method. Consequently, the balance equations $(y 2)$ and $(m 3)$ of (16) contain terms which depend on the frequency. They are written as functions of the dimensionless frequency $\hat{\omega}_{f}$. It is equal to the ratio between $\omega$, the circular frequency, and $\omega_{f 1}$, the circular frequency of the first bending mode of the floors with two fixed ends. Moreover, the natural frequencies of an Euler-Bernoulli beam that is fixed at both extremities are the solutions of the following equation:

$$
2 f_{o}(\hat{\omega}) f_{e}(\hat{\omega})=1-\cos \left(\frac{3 \pi}{2} \sqrt{\hat{\omega}}\right) \cosh \left(\frac{3 \pi}{2} \sqrt{\hat{\omega}}\right)=0
$$

where the function $f_{o}$ vanishes at the frequencies of the odd bending modes and the function $f_{e}$ vanishes at the frequencies of the even bending modes.

In Eq. ( $y 2)$ of (16), the local resonance modifies the inertial term. The mass of the floors $M_{f}=\bar{\rho}_{f} / \ell_{w}$ is multiplied by a frequency dependent function $f$ giving an effective mass. The variations of the function $f$ according to the frequency are plotted in figure 8. The limit of $f$ at very low frequencies is 1 , which means that the effective mass is equal to the real mass as expected. At most of the frequencies higher than $\omega_{f 1}, f\left(\hat{\omega}_{f}\right)$ is between 0 and 1 and the floors seem lighter. But close to the frequencies of the odd bending modes, $f\left(\hat{\omega}_{f}\right)$ becomes infinite and changes its sign. Such an atypical behavior is not observed close to the frequencies of the even bending modes.

The physical origin of the effective mass is the motion of the floors relative to the nodes. By definition the kinematic descriptors correspond to the motion of the nodes. At low frequencies, when every element has a quasi-static behavior at the local scale, all the points of a unit cell experience almost the same motion. At the leading order, the sum of the inertia forces acting on the whole cell is 


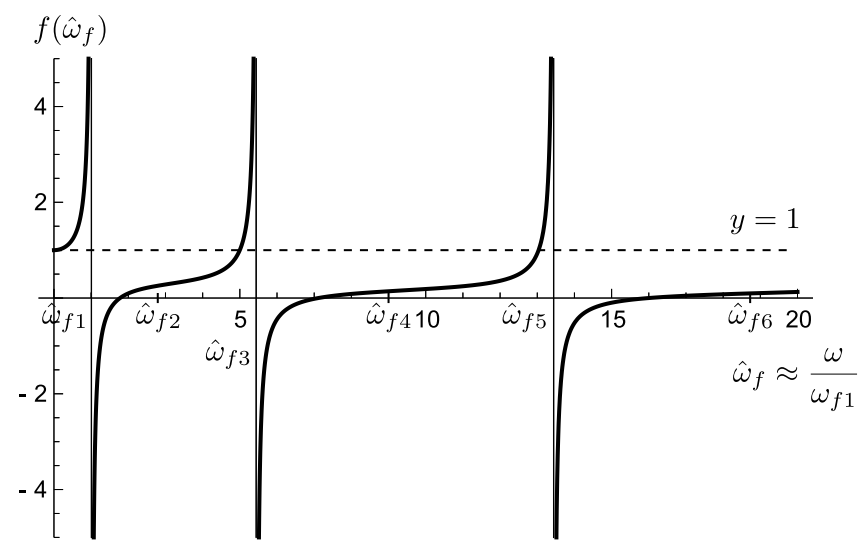

Figure 8: Variations of the function $f$ according to the dimensionless frequency therefore equal to the real mass of the cell multiplied by the acceleration of the nodes. When the floors are in resonance in bending, their motion can strongly differ from the one of the nodes and the inertia forces are modified. Since the bending of the floors generates vertical motions, only the vertical component of the inertia forces is affected by the local resonance. Equation $(y 2)$ of (16) describing the balance of momentum in the $y$ direction contains the effective mass, whereas Eq. (x 1$)$ of (16) describing the balance of momentum in the $x$ direction contains the real mass. During the propagation of macroscopic waves, neighboring nodes undergo almost the same motion because of the scale separation. Such boundary conditions excite the odd bending modes of the floors but not the even bending modes, therefore the effective mass has very different properties in the two situations. For a more detailed presentation of the properties of the effective mass and its consequences, the reader is referred to $[17]$.

In SC materials, the mass is degenerate at the leading order because the mass of the floors is negligible. The effective mass only appears in the higher order equations and the resonance of the floors seems to have almost no effect on the wave propagation. However, the effective mass becomes infinite close to the frequencies of the odd bending modes of the floors. In this case, the 
normalization is no longer valid and the mass of the floors should be taken into account. This can sometimes lead to atypical behaviors at the resonant frequencies and it is important to examine the higher order equations in presence of resonance. Here, the properties of the waves are determined by the balance of momentum in the $x$ direction which is not affected even if we consider the higher orders. Moreover, in the balance of momentum in the $y$ direction [in particular in Eq. (y2) of (16)], the effective mass of the floors is multiplied by the vertical displacement $\tilde{U}_{y}^{0}$ which is always null. All this confirms that the wave propagation is not affected by the local resonance in this case. There is no dispersion and no frequency bandgaps contrary to [17].

Finally, we have to consider the balance of moment of momentum. These equations describe the inner equilibrium of the unit cell and can impose additional kinematic conditions. Contrary to the previous sections, they now contain inertial terms and the equality of $\tilde{\tau}_{x y}^{4}$ and $\tilde{\tau}_{y x}^{4}$ as defined in Eqs. (6) is lost. First, the leading orders of $\tau_{x y}$ and $\tau_{y x}$ are different. In Eq. ( $\left.m 2\right)$ of (16), $\tilde{\tau}_{x y}^{3}$ is balanced by an inertial term and is therefore the first non-zero term for $\tau_{x y}$. Second, $\tilde{\tau}_{y x}^{4}$ is replaced in Eq. $(m 3)$ of $(16)$ by $\tau\left(\hat{\omega}_{f}\right)$ the expression of which is given in Eq. (17e). This term combines elastic effects and inertial effects which cannot be separated. Its limit at low frequencies is the term $\tilde{\tau}_{y x}^{4}$ defined in Eqs. (6). The term $\tau\left(\hat{\omega}_{f}\right)$ appears in Eq. (m3) of (16) but its normalization is not valid close to the frequencies of the even bending modes of the floors. At these frequencies, $f_{e}\left(\hat{\omega}_{f}\right)=0$ and $\tau\left(\hat{\omega}_{f}\right)$ becomes infinite. This prevents the nodes from rotating and the phase difference between the compressional waves in the floors is impossible. This behavior is due to the fact that the boundary conditions of a beam are not independent at its natural frequencies. 


\section{Tension-compression of the walls and bending resonance of the floors}

\subsection{Leading order and wave propagation}

To observe waves generated by the last mechanism identified in Section 3, the tension-compression of the walls, the circular frequency has to be increased up to $O\left(\omega_{r}\right)$. As these frequencies are higher than the first resonant frequencies in bending of the floors, the bending wavelength in these elements $\lambda_{b f}$ is smaller than their length $\ell_{f}$. Nevertheless, $\lambda_{b f}$ remains much greater than their thickness $a_{f}$ [more precisely $\left.a_{f}=O\left(\epsilon^{5 / 4} \lambda_{b f}\right)\right]$ and the Euler-Bernoulli beam model is still valid. As a result, we can use the HPDM method but we have to keep the complete expressions of the shear force and the bending moment in the floors as in Section 6. For the three other mechanisms (tension-compression of the floors, bending and tension-compression of the walls), the wavelengths generated in the elements are much greater than their length. The associated forces and moments can be expanded normally. Note that the walls reach the dynamic regime for tension-compression without being in resonance in bending.

It is also necessary to modify the asymptotic expansions of the nodal kinematic descriptors. Owing to the local resonance in bending of the floors, some inertial terms depend on the effective mass and, consequently, on the function $f\left(\hat{\omega}_{f}\right)$ defined in Eq. (17b). For the studied frequencies, the order of magnitude of the function is $f\left(\hat{\omega}_{f}\right)=O\left(\epsilon^{1 / 4}\right)$. Therefore, the nodal descriptors have to be expanded according to the powers of $\epsilon^{1 / 4}$ instead of $\epsilon$ for all the inertial terms to be balanced by an elastic term [Eq. (3)]. Having 1/4 for the order of magnitude of $f\left(\hat{\omega}_{f}\right)$ means that, when the frequency increases, the inertial terms increase more slowly in situations with local resonance. The physical explanation of this phenomenon is that the higher modes of the elements in resonance have more anti-nodes vibrating in opposite directions. Consequently the proportion of the mass that moves in phase with the nodes of the unit cell is smaller than in 
situations with a quasi-static behavior at the local scale. It is consistent with the fact that the effective mass is smaller than the real mass at most of the frequencies higher than the frequency of the first bending mode (see figure 8 and $[17])$.

With these modifications, the HPDM method provides the following equations for the balance of momentum.

$$
\begin{array}{lr}
M_{w} \omega^{2} \tilde{U}_{x}^{0}=0 & (x-1) \\
E_{y} \frac{\partial^{2} \tilde{U}_{y}^{0}}{\partial y^{2}}+M_{w} \omega^{2} \tilde{U}_{y}^{0}=0 & (y 0) \\
G_{w}\left(\tilde{\theta}^{0}+\frac{\partial \tilde{U}_{x}^{0}}{\partial y}\right)=0 & (m 0)
\end{array}
$$

Because of the increase of the frequency, the inertial term in the balance equation in the $x$ direction is greater than the elastic terms. This is the reason why it appears at the order -1 . Consequently, we have $\tilde{U}_{x}^{0}=0$ and Eq. $(m 0)$ imposes $\tilde{\theta}^{0}=0$.

The wave propagation is described by the balance of momentum in the $y$ direction. The introduction of the expression (8) of the displacement field in Eq. $(y 0)$ gives the wave number:

$$
k(\alpha)=\frac{\omega}{|\sin (\alpha)|} \sqrt{\frac{M_{w}}{E_{y}}} \Rightarrow c(\alpha)=|\sin (\alpha)| \sqrt{\frac{E_{y}}{M_{w}}}
$$

The macroscopic waves are polarized in the direction of the walls and can travel in all the directions except in the $x$ direction $[\sin (\alpha)=0]$. The speed depends on the direction of propagation but not on the frequency. It is maximal for the waves traveling in the $y$ direction. This description corresponds to the Y-mode of [17].

The roles of the floors and the walls are reversed compared with Section 6.1. The macroscopic waves are generated by compressional waves which travel in the walls and which can be out-of-phase. This mechanism is described in figure 9 with the same notation as in figure 7 . The horizontal black sinusoids at the 


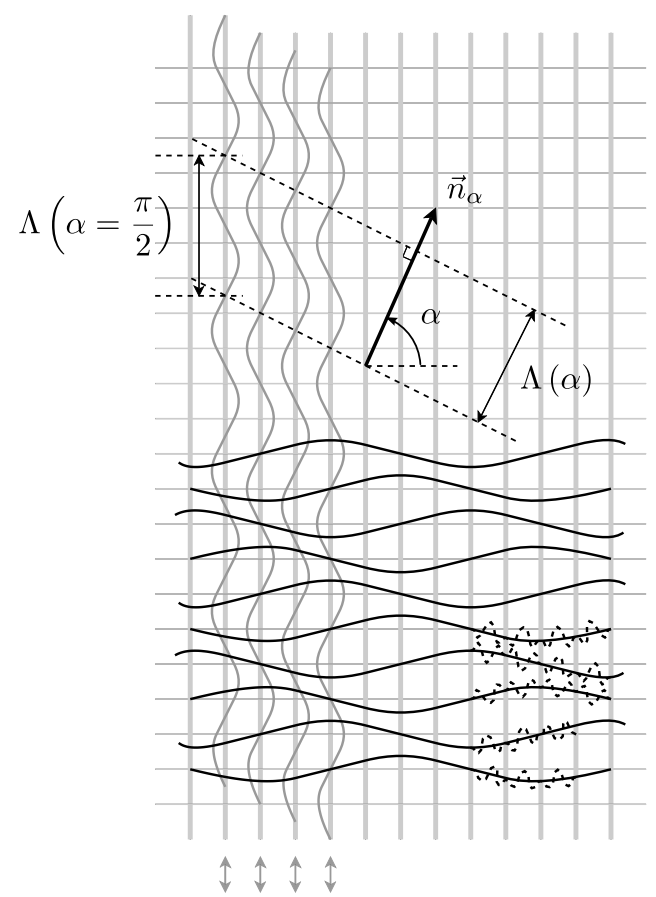

Figure 9: Macroscopic shear-compression wave traveling in the direction $\vec{n}_{\alpha}$ with the Y-mode 
bottom of the figure represent the deformation of the floors caused by the phase difference between the compressional waves in the walls. The total deformation of the floors is obtained by adding the deflection induced by the resonance in bending (drawn with dotted lines in the bottom right corner).

\subsection{Higher orders and local resonance}

At the leading order, the speed only depends on the real mass of the walls and the resonance of the floors seems to have no effect on the wave propagation. However the situation is not the same as in Section 6. Now the properties of the waves are given by the balance equations in the $y$ direction and the higher order equations probably contain the effective mass of the floors. To determine if there are atypical behaviors at the resonant frequencies, we have to analyze the higher orders. Due to the expansions according to the powers of $\epsilon^{1 / 4}$, there are more equations than in the previous sections. Only the equations with new terms are written afterwards. Moreover, the equations of lower order and the equations describing the balance of moment of momentum are used to reduce 
the other equations.

$$
\begin{aligned}
& \left\{\begin{array}{l}
M_{w} \omega^{2} \tilde{U}_{x}^{0}=0 \\
M_{w} \omega^{2} \tilde{U}_{x}^{1}+E_{x} \frac{\partial^{2} \tilde{U}_{x}^{0}}{\partial x^{2}}+M_{f} \omega^{2} \tilde{U}_{x}^{0}+\frac{\left(\ell_{w} M_{w} \omega^{2}\right)^{2}}{60 G_{w}} \tilde{U}_{x}^{0}=0
\end{array}\right. \\
& (x-1) \\
& G_{w}\left(\tilde{\theta}^{1}+\frac{\partial \tilde{U}_{x}^{1}}{\partial y}\right)+\frac{\ell_{w}^{2}}{15} M_{w} \omega^{2} \frac{\partial \tilde{U}_{x}^{0}}{\partial y}=0 \\
& G_{w}\left(\tilde{\theta}^{2}+\frac{\partial \tilde{U}_{x}^{2}}{\partial y}\right)+\frac{\ell_{w}^{2}}{15} M_{w} \omega^{2} \frac{\partial \tilde{U}_{x}^{1}}{\partial y}+\frac{11 \ell_{w}^{4}\left(M_{w} \omega^{2}\right)^{2}}{6300 G_{w}} \frac{\partial \tilde{U}_{x}^{0}}{\partial y}=0 \\
& G_{w}\left(\tilde{\theta}^{5 / 2}+\frac{\partial \tilde{U}_{x}^{5 / 2}}{\partial y}\right)+\frac{\ell_{w}^{2}}{15} M_{w} \omega^{2} \frac{\partial \tilde{U}_{x}^{3 / 2}}{\partial y}+\frac{11 \ell_{w}^{4}\left(M_{w} \omega^{2}\right)^{2}}{6300 G_{w}} \frac{\partial \tilde{U}_{x}^{1 / 2}}{\partial y} \\
& -\frac{3 \pi^{2} G_{f} \hat{\omega}_{f}}{16 f_{e}\left(\hat{\omega}_{f}\right) f_{o}\left(\hat{\omega}_{f}\right)}\left(\cosh \left(\frac{3 \pi}{2} \sqrt{\hat{\omega}_{f}}\right)-\cos \left(\frac{3 \pi}{2} \sqrt{\hat{\omega}_{f}}\right)\right) \frac{\partial \tilde{U}_{y}^{0}}{\partial x}=0
\end{aligned}
$$

The functions $f, f_{o}$ and $f_{e}$ are defined in Eqs. (17).

As expected the effective mass of the floors appears in Eq. $\left(y \frac{5}{4}\right)$ and the correctors probably depend on the frequency. There is dispersion but its effects are negligible at most of the frequencies (figure 10). Nevertheless, the estimation of the effective mass is not valid in the neighborhood of the frequencies of the odd bending modes of the floors which are the poles of the function $f\left(\hat{\omega}_{f}\right)$. At these frequencies, it becomes infinite and a rigorous approach would require to take into account its effects at the leading order. The neighborhood of the frequencies of the odd bending modes of the floors certainly corresponds to frequency bandgaps as in [17]. Thus, the validity domain of the leading order is provided by the higher orders. Here the discrete frequencies of the odd bending 


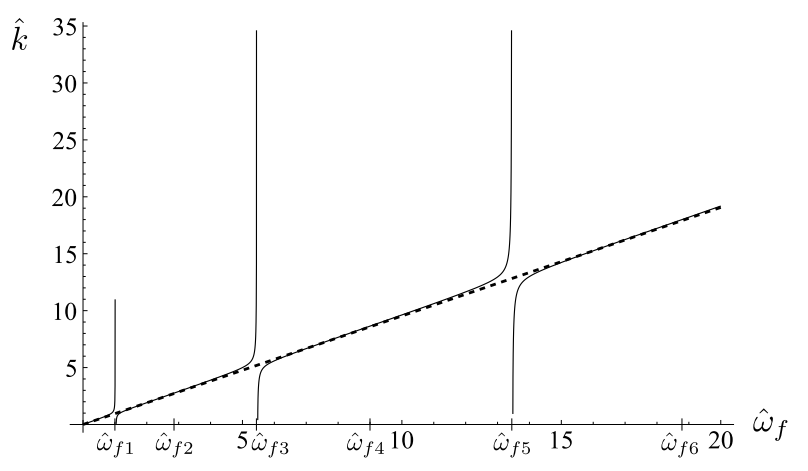

Figure 10: Variation of the dimensionless wave number $\hat{k}=\frac{k(\omega)}{\omega_{f 1}} \sqrt{\frac{E_{y}}{M_{w}+M_{f}}}$ according to the dimensionless frequency $\hat{\omega}_{f} \approx \frac{\omega}{\omega_{f 1}}$ for the Y-mode with $\alpha=\pi / 2$ and $M_{f}=M_{w} / 10$. The dotted line corresponds to the leading order described by Eq. (19). For the continuous line $M_{w}$ is replaced by $M_{w}+M_{f} f\left(\hat{\omega}_{f}\right)$

modes of the floors have to be excluded.

Finally, we have to examine the kinematic conditions imposed by the inner equilibrium of the cell. As the first orders of the horizontal displacement $U_{x}$ are equal to zero, the inertial terms in the balance of moment of momentum are null. The first non-zero term for $\tau_{x y}$, which is defined in Eqs. (6) and is associated with the local bending of the walls, is therefore $\tilde{\tau}_{x y}^{7 / 2}$ in Eq. $\left(m \frac{5}{2}\right)$. Concerning the local bending of the floors, neither $\tau_{y x}$ [Eqs. (6)] nor the function $\hat{\tau}\left(\hat{\omega}_{f}\right)$ [Eq.(17e)] exists. The particularity of this case is that the two terms constituting $\hat{\tau}\left(\hat{\omega}_{f}\right)$ have different orders of magnitude. Consequently, they appear in two different equations. The first part, which is proportional to $\partial_{x} \tilde{U}_{y}^{0}$, is in Eq. $\left(m \frac{5}{2}\right)$ and it balances $\tilde{\tau}_{x y}^{7 / 2}$. Its coefficient becomes infinite in the neighborhood of the even bending modes of the floors $\left[f_{e}\left(\hat{\omega}_{f}\right)=0\right]$. This prevents the phase difference between the compressional waves traveling in the walls as in Section 6 . 


\section{Behavior of real materials}

\subsection{Summary of the main results}

In the previous sections, four types of waves with different kinematics were identified. They are generated by four different mechanisms: the local bending of the floors, the propagation of synchronized bending waves in the walls, the propagation of possibly out-of-phase compressional waves in the floors, the propagation of possibly out-of-phase compressional waves in the walls. The resulting properties of the macroscopic waves are summarized in Tab. 1.

\begin{tabular}{|c|c|}
\hline Normalization & $\frac{a_{w}}{\ell_{w}}=O\left(\epsilon^{1 / 2}\right), \quad \frac{a_{f}}{\ell_{w}}=O\left(\epsilon^{3 / 2}\right)$ \\
\hline$\omega=O\left(\epsilon^{2} \omega_{r}\right)$ & $\begin{array}{l}\text { - Propagation in the } x \text { direction } \\
\text { - Shear waves generated by the local bending of the } \\
\text { floors } \\
\text { - No dispersion } \\
\qquad c=\sqrt{G_{f} / M_{w}}\end{array}$ \\
\hline$\omega=O\left(\epsilon^{3 / 2} \omega_{r}\right)$ & $\begin{array}{l}\text { - Propagation in the } y \text { direction } \\
\text { - Shear waves generated by bending waves in the } \\
\text { walls } \\
\text { - Dispersion } \\
\qquad c=\sqrt[4]{\omega^{2} E I_{z} / M_{w}}\end{array}$ \\
\hline
\end{tabular}




\begin{tabular}{|c|c|}
\hline Normalization & $\frac{a_{w}}{\ell_{w}}=O\left(\epsilon^{1 / 2}\right), \quad \frac{a_{f}}{\ell_{w}}=O\left(\epsilon^{3 / 2}\right)$ \\
\hline$\omega=O\left(\epsilon^{1 / 2} \omega_{r}\right)$ & $\begin{array}{l}\text { - Propagation in all the directions except in the } y \\
\text { direction } \\
\text { - Shear-compression waves polarized in the } x \text { di- } \\
\text { rection generated by compressional waves in the } \\
\text { floors (X-mode) } \\
\text { - Almost no effect of the bending resonance of the } \\
\text { floors } \\
\text { - No dispersion } \\
\qquad c=|\cos (\alpha)| \sqrt{E_{x} / M_{w}}\end{array}$ \\
\hline$\omega=O\left(\omega_{r}\right)$ & $\begin{array}{l}\text { - Propagation in all the directions except in the } x \\
\text { direction } \\
\text { - Shear-compression waves polarized in the } y \text { direc- } \\
\text { tion generated by compressional waves in the walls } \\
\text { (Y-mode) } \\
\text { - Negligible dispersion (effective mass of the floors } \\
\text { in correctors) and frequency bandgaps because of } \\
\text { the bending resonance of the floors } \\
\qquad c=|\sin (\alpha)| \sqrt{\frac{E_{y}}{M_{w}}} \quad \text { (leading order) }\end{array}$ \\
\hline
\end{tabular}

Table 1: Waves in SC materials [figure 1(b)]

Frame materials are known for being strongly anisotropic [42, 17] because waves are channeled in the two directions of the elements. The anisotropy is even more marked for SC materials. On the one hand, the macroscopic shear waves in the direction of the floors and in the direction of the walls are 
generated by two different mechanisms. The existence of a stiffness contrast enables the walls to deform in bending at the macroscopic scale. Consequently, the equivalent continuum is a second grade medium with an inner moment as it will be explained in Section 9. There is dispersion for the shear waves propagating in the vertical direction. On the other hand, the elastic moduli in the two directions of the elements have different orders of magnitude. The waves generated by the tension-compression of the floors (X-mode) and those generated by the tension-compression of the walls (Y-mode) appear therefore in different frequency ranges. The stiffness contrast was introduced by modifying the thickness of the elements. Since the walls and the floors are made of the same material, this also creates a mass contrast. The mass of the floors appears in the higher order equations and the bending resonance of the floors has a smaller effect on the wave propagation than in SP materials.

To make the comparison between SC and SP materials easier the main results of [17] are given in Tab. 2. In this work, the HPDM method was used to study the wave propagation in a frame material with similar walls and floors. Such a material has the same behavior in the two directions of the elements. The walls cannot deform in bending at the macroscopic scale. As a result, all the shear waves are generated by the local bending. They occur in the same frequency range and there is no dispersion. Likewise, the $\mathrm{X}$-mode and the Y-mode of the shear-compression waves travel in the same frequency range. In presence of local resonance, the effective mass of the elements appears at the leading order. There is dispersion in addition to frequency bandgaps.

Thus frame materials can exhibit two types of behaviors. Either the equivalent continuum is a "classical" medium and shear waves travel in the two directions of the elements without dispersion. Or the equivalent continuum is a second grade medium and there is dispersion for the shear waves in one direction. If we now consider a real material with given properties, what is its behavior? The only difference between the two models is the stiffness contrast 


\begin{tabular}{|c|c|}
\hline Normalization & $\frac{a_{w}}{\ell_{w}}=O(\epsilon), \quad \frac{a_{f}}{\ell_{w}}=O(\epsilon)$ \\
\hline$\omega=O\left(\epsilon \omega_{r}\right)$ & $\begin{array}{l}\text { - Propagation in the } x \text { and } y \text { directions } \\
\text { - Shear waves generated by the local bending of the } \\
\text { elements } \\
\text { - No dispersion } \\
\qquad c=\sqrt{G /\left(M_{w}+M_{f}\right)}\end{array}$ \\
\hline$\omega=O\left(\omega_{r}\right)$ & $\begin{array}{l}\text { - Propagation in all the directions except in the } y \\
\text { direction for the waves polarized in the } x \text { direction } \\
\text { (X-mode) and propagation in all the directions ex- } \\
\text { cept in the } x \text { direction for the waves polarized in } \\
\text { the } y \text { direction (Y-mode) } \\
\text { - Shear-compression waves generated by compres- } \\
\text { sional waves in the floors for the X-mode and by } \\
\text { compressional waves in the walls for the Y-mode } \\
\text { - Bending resonance of the elements: dispersion (ef- } \\
\text { fective mass at the leading order) and frequency } \\
\text { bandgaps } \\
\quad c=|\cos (\alpha)| \sqrt{\frac{E_{x}}{M_{w} f\left(\hat{\omega}_{w}\right)+M_{f}}} \\
\quad c=|\sin (\alpha)| \sqrt{\frac{E_{y}}{M_{w}+M_{f} f\left(\hat{\omega}_{f}\right)}} \\
\text { Y-mode: }\end{array}$ \\
\hline
\end{tabular}

Table 2: Waves in SP materials [figure 1(a)] 
introduced during the normalization. As this step makes an extensive use of the scale ratio $\epsilon$, we begin with important comments about this parameter. Then, a criterion is built for the identification of the behavior without performing homogenization. Finally, we explain how to determine the effects of the local resonance.

\subsection{The scale ratio}

To clearly distinguish the physical scale ratio from the mathematical small parameter used in homogenization, the physical scale ratio will be written $\tilde{\epsilon}$ afterwards as in [29]. It corresponds to the ratio between $\ell_{c}$, the characteristic length of the unit cell and $L$, the characteristic length of the deformation of the frame material: $\tilde{\epsilon}=\ell_{c} / L$. As both lengths are finite, the scale ratio is a finite quantity. If the condition of scale separation is respected $(\tilde{\epsilon}<<1)$, it is possible to derive an equivalent continuum by homogenization. As mentioned above, the physics of the problem is introduced in the model during the normalization. The powers of $\tilde{\epsilon}$ are used as a kind of "unit of measurement" to convert the numerical values of the physical parameters into orders of magnitude. Then, homogenization consists in replacing the physical $\tilde{\epsilon}$ by a mathematical $\epsilon$ which is made to approach zero. Thanks to the normalization, the relative orders of magnitude of the physical terms are kept identical from the real frame material to the continuum obtained at the limit. However, as the physical scale ratio $\tilde{\epsilon}$ is a finite quantity, the homogenized model is an approximation of the behavior of the real material. By only considering the leading order as in the previous sections, the order of magnitude of the neglected correctors is $O(\tilde{\epsilon})$.

A reliable estimation of the scale ratio $\tilde{\epsilon}$ is therefore needed to identify the behavior of a given frame material and to evaluate the accuracy of the equivalent continuum. The size of the unit cell $\ell_{c}=\ell_{w}$ is fixed but the macroscopic length $L$ depends on the external actions. In the case of wave propagation, it can be 
shown [43] that:

$$
L=\frac{\Lambda}{2 \pi} \quad \Rightarrow \quad \tilde{\epsilon}=\frac{2 \pi \ell_{c}}{\Lambda}=\ell_{c} k
$$

where $\Lambda$ is the macroscopic wavelength and $k$ the wave number. Equation (21) is very useful for the estimation of the accuracy but it is not convenient for the determination of the appropriate model. The main problem is that it is necessary to know the model to calculate the wavelength. This is the reason why the next section proposes another approach for the identification of the behavior of a real material.

The expression given in Eq. (21) also shows that the scale ratio $\tilde{\epsilon}$ depends on the frequency. When $\omega$ increases, $\Lambda$ decreases and $\tilde{\epsilon}$ increases. As the physical parameters have fixed values, the orders of magnitude provided by the normalization are modified: the contrasts become greater. For some frame materials, this can lead to a change of the homogenized model. Moreover, the accuracy of the equivalent continuum is related to the value of $\tilde{\epsilon}$ and the model is valid in a limited frequency range. The highest frequency is imposed by the condition of scale separation. The lowest frequency depends on the specimen size $H$. When $L$ becomes greater than $H$, the material has a quasi-static behavior. Thus, dynamic homogenization is restricted to the following domain:

$$
\ell_{c}<<L<H
$$

Finally, note that the expressions of $\Lambda$ and $L$ change with the nature of the macroscopic waves. The homogenizable domain is different in each case. The waves generated by more rigid mechanisms appear at higher frequencies but the domains of different waves can overlap.

\subsection{Model identification}

To build a criterion to determine whether a given frame material behaves as a second grade or a "classical" medium, we consider here the intermediate situation. After designing the appropriate geometry, we derive by homogenization 
the richest model with both types of bending and we study how it degenerates.

In balance equations, the term describing the macroscopic bending is the fourth derivative [see for example Eq. ( $x 2)$ of (4)]:

$$
-E I_{z} \frac{\partial^{4} \tilde{U}_{x}^{0}}{\partial y^{4}}=O\left(\frac{E_{w} I_{w}}{\ell_{f}} \frac{\tilde{U}_{x}^{0}}{L^{4}}\right)
$$

according to the expression of $E I_{z}$ given in Section 3. The most general term corresponding to the local bending comes from the balance equations of SP materials [17]:

$$
\begin{gathered}
G\left(\frac{\partial^{2} \tilde{U}_{y}^{0}}{\partial y \partial x}+\frac{\partial^{2} \tilde{U}_{x}^{0}}{\partial y^{2}}\right)=O\left(G \frac{\tilde{U}_{x}^{0}}{L^{2}}\right) \\
\text { with } \quad \frac{1}{G}=\frac{1}{G_{f}}+\frac{1}{G_{w}}
\end{gathered}
$$

The moduli $G_{f}$ and $G_{w}$ are also defined in Section 3. The moduli $E I_{z}$ and $G_{w}$ are related to the bending of the walls at different scales. Consequently the contrast between $E I_{z}$ and $G_{w}$ is fixed:

$$
\frac{E I_{z}}{G_{w} L^{2}}=\frac{E_{w} I_{w}}{\ell_{f}} \frac{\ell_{w}^{2} \ell_{f}}{12 E_{w} I_{w}} \frac{1}{L^{2}}=O\left(\tilde{\epsilon}^{2}\right)
$$

When the two types of bending have the same importance, the terms (23) and (24) have the same order of magnitude. Because of Eq. (25), the only possibility is that the floors are more flexible than the walls and the shear modulus $G$ degenerates into $G_{f}$ as in Eq. (7).

$$
\begin{aligned}
G\left(\frac{\partial^{2} \tilde{U}_{y}^{0}}{\partial y \partial x}+\frac{\partial^{2} \tilde{U}_{x}^{0}}{\partial y^{2}}\right) & =O\left[G_{f}\left(\frac{\partial^{2} \tilde{U}_{y}^{0}}{\partial y \partial x}+\frac{\partial^{2} \tilde{U}_{x}^{0}}{\partial y^{2}}\right)\right] \\
& =O\left[\frac{E_{f} I_{f}}{\ell_{w} \ell_{f}^{2}} \frac{\tilde{U}_{x}^{0}}{L^{2}}\right]
\end{aligned}
$$

By assuming that the walls and the floors are made of similar materials as in Eqs. (2a) and that only the thicknesses are significantly different, we obtain the following condition between $e_{f}$ and $e_{w}$, the orders of magnitude of the 
thicknesses of the floors and the walls respectively.

$$
\begin{aligned}
& \frac{E_{w} I_{w}}{\ell_{f}} \frac{\tilde{U}_{x}^{0}}{L^{4}}=O\left(\frac{E_{f} I_{f}}{\ell_{w} \ell_{f}^{2}} \frac{\tilde{U}_{x}^{0}}{L^{2}}\right) \Rightarrow \quad \frac{I_{f}}{I_{w}}=O\left(\frac{\ell_{w} \ell_{f}}{L^{2}}\right) \\
& \Rightarrow\left(\frac{a_{f}}{a_{w}}\right)^{3}=O\left(\epsilon^{2}\right) \\
& \Rightarrow 3\left(e_{f}-e_{w}\right)=2 \text { with } \frac{a_{f}}{\ell_{w}}=O\left(\tilde{\epsilon}^{e_{f}}\right), \quad \frac{a_{w}}{\ell_{w}}=O\left(\tilde{\epsilon}^{e_{w}}\right)
\end{aligned}
$$

This relation was not verified for the two frame materials previously studied (Tabs. 1 and 2). There was therefore only one governing mechanism at the leading order for the shear waves in the direction of the walls.

We now homogenize a frame material respecting the condition (26) to derive the general model. The normalization is given by Eqs. (2a) and (27).

$$
\frac{a_{w}}{\ell_{w}}=O(\tilde{\epsilon}) \quad \frac{a_{f}}{\ell_{w}}=O\left(\tilde{\epsilon}^{5 / 3}\right)
$$

In that case, the macroscopic shear waves occur at frequencies such that $\omega=O\left(\tilde{\epsilon}^{2} \omega_{r}\right)$. Since the order of magnitude of the ratio $a_{f} / a_{w}$ is not an integer, the kinematic descriptors must now be expanded according to the powers of $\epsilon^{1 / 3}$ :

$$
\begin{aligned}
X(\epsilon, x, y)=X^{0}(x, y)+ & \epsilon^{1 / 3} X^{1 / 3}(x, y)+\epsilon^{2 / 3} X^{2 / 3}(x, y) \\
& +\epsilon X^{1}(x, y)+\epsilon^{4 / 3} X^{4 / 3}(x, y)+\epsilon^{5 / 3} X^{5 / 3}(x, y)+\ldots
\end{aligned}
$$

where $X$ stands for $U_{x}, U_{y}$ or $\theta$. Then, the HPDM method provides the following equations for the balance of momentum. Only the equations with new terms are written afterwards and the equations describing the balance of moment of 
momentum were used to reduce the other equations.

$$
\begin{aligned}
& \left\{\begin{array}{l}
E_{x} \frac{\partial^{2} \tilde{U}_{x}^{0}}{\partial x^{2}}=0 \\
E_{x} \frac{\partial^{2} \tilde{U}_{x}^{10 / 3}}{\partial x^{2}}+G_{f}\left(\frac{\partial^{2} \tilde{U}_{x}^{0}}{\partial y^{2}}+\frac{\partial^{2} \tilde{U}_{y}^{0}}{\partial y \partial x}\right)-E I_{z} \frac{\partial^{4} \tilde{U}_{x}^{0}}{\partial y^{4}}+M_{w} \omega^{2} \tilde{U}_{x}^{0}=0
\end{array}\right. \\
& \left\{\begin{array}{l}
E_{y} \frac{\partial^{2} \tilde{U}_{y}^{0}}{\partial y^{2}}=0 \\
E_{y} \frac{\partial^{2} \tilde{U}_{y}^{4}}{\partial y^{2}}+G_{f}\left(\frac{\partial^{2} \tilde{U}_{x}^{0}}{\partial x \partial y}+\frac{\partial^{2} \tilde{U}_{y}^{0}}{\partial x^{2}}\right)+M_{w} \omega^{2} \tilde{U}_{y}^{0}=0
\end{array}\right. \\
& \left\{\begin{array}{l}
G_{w}\left(\tilde{\theta}^{0}+\frac{\partial \tilde{U}_{x}^{0}}{\partial y}\right)=0 \\
G_{w}\left(\tilde{\theta}^{2}+\frac{\partial \tilde{U}_{x}^{2}}{\partial y}\right)-G_{f}\left(-\tilde{\theta}^{0}+\frac{\partial \tilde{U}_{y}^{0}}{\partial x}\right)=0
\end{array}\right.
\end{aligned}
$$

As expected the terms associated with the local bending and the macroscopic bending appear in the same equation [Eq. $\left.\left(x \frac{10}{3}\right)\right]$. The introduction of the expression (8) of the displacement field shows that macroscopic plane waves can travel in the two directions of the elements. The shear waves in the $x$ direction are generated by the local bending of the floors as in Section 4 and their speed is given by Eq. (10). The waves in the $y$ direction are also shear waves and the wave number is deduced from Eq. $\left(x \frac{10}{3}\right)$.

$$
k^{2}=\frac{G_{f}}{2 E I_{z}}\left(\sqrt{1+4 M_{w} \omega^{2} \frac{E I_{z}}{G_{f}^{2}}}-1\right)
$$

To obtain a model which generalizes both the descriptions of Tabs. 1 and 2, $G_{f}$ is replaced by the shear modulus $G$ and $M_{w}$ by the total mass $M_{s}=M_{w}+M_{f}$. Since the contributions of $G_{w}$ and $M_{f}$ are negligible for the studied frame material, this modification has almost no effect on the wave number.

$$
k^{2}=\frac{G}{2 E I_{z}}\left(\sqrt{1+4 M_{s} \omega^{2} \frac{E I_{z}}{G^{2}}}-1\right)
$$

This model combines the two mechanisms: the shear of the cell generated by the local bending of the elements and the macroscopic bending. It can degenerate 
into the other models depending on the order of magnitude of the term under the square root in Eq. (29).

General model with dispersion:

$$
4 M_{s} \omega^{2} \frac{E I_{z}}{G^{2}}=O(1) \quad \Rightarrow \quad k^{2}=\frac{G}{2 E I_{z}}\left(\sqrt{1+4 M_{s} \omega^{2} \frac{E I_{z}}{G^{2}}}-1\right)
$$

Macroscopic bending and dispersion:

$$
4 M_{s} \omega^{2} \frac{E I_{z}}{G^{2}}>>(1) \quad \Rightarrow \quad k^{2}=\sqrt{\frac{M_{s} \omega^{2}}{E I_{z}}}
$$

Shear of the cell and no dispersion:

$$
4 M_{s} \omega^{2} \frac{E I_{z}}{G^{2}}<<O(1) \quad \Rightarrow \quad k^{2}=\frac{M_{s} \omega^{2}}{G}
$$

This provides a dimensionless criterion to identify the behavior of a given frame material. The governing mechanism is the most rigid as in a connection in parallel. Note that the criterion directly compares the stiffnesses of the two types of bending and not the thicknesses of the elements. Since it is completely analytic, it is possible to investigate the role of every cell parameter: the geometry of the elements and the material properties. The criterion also depends on the frequency. The same frame material can behave as a "classical" medium at low frequencies and as a second grade medium at high frequencies. During the propagation of bending waves, the walls experience deformations on a lengthscale related to the wavelength. When the frequency increases, the wavelength decreases and the macroscopic bending becomes more rigid.

A similar criterion for the macroscopic bending of the floors is obtained by changing the definition of the modulus of the macroscopic bending: $E I_{z}=E_{f} I_{f} / \ell_{w}$.

\subsection{Effects of the local resonance}

After analyzing the properties of the shear waves, we consider here the properties of the shear-compression waves. As illustrated in Tabs. 1 and 2, these waves can 
be more or less affected by the local resonance in bending. Their study includes three steps.

First, we have to determine whether the local resonance occurs in the same frequency range as the wave propagation. The domain of the shear-compression waves is estimated thanks to the condition (22) by neglecting the local resonance. The real mass of the elements is used in the calculation of the macroscopic length $L$ :

$$
\left\{\begin{array}{l}
L=\frac{1}{k}=\frac{|\cos (\alpha)|}{\omega} \sqrt{\frac{E_{x}}{M_{s}}} \text { for the X-mode } \\
L=\frac{1}{k}=\frac{|\sin (\alpha)|}{\omega} \sqrt{\frac{E_{y}}{M_{s}}} \text { for the Y-mode }
\end{array}\right.
$$

with $M_{s}=M_{w}+M_{f}$. As explained in Appendix and [17], there is a quasi-static state at the local scale as long as $2 \pi \ell / \lambda_{b}<<1$ where $\ell$ is the length of an element and $\lambda_{b}$ is the bending wavelength in that element. If this condition is respected for the walls and the floors in the whole domain of the shear-compression waves, there is no bending resonance. The speed of the macroscopic waves only varies with their polarization and their direction of propagation.

If their is resonance, its effect depends on which elements are affected. The shear-compression waves are generated by the propagation of compressional waves in the elements. When only the elements deformed by the compressional waves are in resonance as in Section 6, the equations describing the macroscopic waves contain the real mass of the elements even at the higher orders. The local resonance has almost no effect. It imposes kinematic conditions close to the frequencies of the even bending modes of the resonating elements. On the contrary, when the elements orthogonal to the compressional waves are in resonance, the properties of the macroscopic waves depend on their effective mass. In addition to the kinematic conditions, there is dispersion and bandgaps close to the frequencies of the odd bending modes of the resonating elements as in [17]. However, if there is an important mass contrast between the elements, it is possible that the variations of the speed with the frequency are negligible as in Section 7. 


\section{Generic frame continuum}

We now propose to build a generic model which can degenerate into any of the equivalent continua previously derived for frame materials. Such a model is obtained by artificially giving the same order of magnitude to every term. Thus, the tildes and superscripts are removed from the variables. To describe all the mechanisms, the model must include normal stresses, $\sigma_{x x}$ and $\sigma_{y y}$, shear stresses, $\tau_{x y}$ and $\tau_{y x}$, and an inner moment, $\mu_{z}$.

$$
\begin{aligned}
\sigma_{x x} & =E_{x} \frac{\partial U_{x}}{\partial x} & \tau_{x y} & =G_{w}\left(\theta+\frac{\partial U_{x}}{\partial y}\right) \\
\sigma_{y y} & =E_{y} \frac{\partial U_{y}}{\partial y} & \tau_{y x} & =G_{f}\left(-\theta+\frac{\partial U_{y}}{\partial x}\right)
\end{aligned}
$$

If we assume that the walls are the stiffest elements, the expression of the inner moment is:

$$
\mu_{z}=-E I_{z} \frac{\partial^{2} U_{x}}{\partial y^{2}} \quad \text { with } \quad E I_{z}=\frac{E_{w} I_{w}}{\ell_{f}}
$$

The balance of momentum in the $x$ and $y$ directions and the balance of moment of momentum are then:

$$
\begin{aligned}
& \frac{\partial \sigma_{x x}}{\partial x}+\frac{\partial}{\partial y}\left(\tau_{x y}+\frac{\partial \mu_{z}}{\partial y}\right)+\left[M_{w} f\left(\hat{\omega}_{w}\right)+M_{f}\right] \omega^{2} U_{x}=0 \\
& \frac{\partial \sigma_{y y}}{\partial y}+\frac{\partial \tau_{y x}}{\partial x}+\left[M_{w}+M_{f} f\left(\hat{\omega}_{f}\right)\right] \omega^{2} U_{y}=0 \\
& \tau_{x y}-\tau_{y x}+J_{x}(\omega) \omega^{2} \frac{\partial U_{x}}{\partial y}+J_{y}(\omega) \omega^{2} \frac{\partial U_{y}}{\partial x}+J_{\theta}(\omega) \omega^{2} \theta=0
\end{aligned}
$$

The function $f$ is defined in Eq. (17). It describes the variations of the effective mass and its limit is 1 as $\hat{\omega}$ approaches zero. The expression of the dimensionless frequency $\hat{\omega}_{f}$ is given in Eq. (17a). It is equal to the ratio between $\omega$ and $\omega_{f 1}$ the circular frequency of the first bending mode of the floors with two fixed ends. Similarly, $\hat{\omega}_{w}$ is equal to the ratio between $\omega$ and $\omega_{w 1}$ the circular frequency of the first bending mode of the walls with two fixed ends. Its expression is obtained by replacing the properties of the floors by the ones of the walls in Eq. (17a). As in [17], the inertial term in the balance of momentum in the $x$ direction contains the effective mass of the walls and the real mass 
of the floors, whereas the inertial term in the balance of momentum in the $y$ direction contains the real mass of the walls and the effective mass of the floors. The functions $J_{x}, J_{y}$ and $J_{\theta}$ correspond to both the inertial terms which can appear in the balance of moment of momentum [see for instance Eqs. (16) and (20)] and the function $\tau(\hat{\omega})$ which combines the elastic and inertial effects in presence of resonance (see Sections 6.2, 7.2 and [17]). These functions are built in such a way that they vanish when $\omega$ approaches zero.

The balance of moment of momentum describes the inner equilibrium of the unit cell. It is used to eliminate the node rotation $\tilde{\theta}^{0}$ which is a "hidden" degree of freedom as explained in Section 3. As a result, the behavior of the generic frame continuum is described by the usual translational degrees of freedom and the balance equations in the $x$ and $y$ directions. But, contrary to the classical continuum mechanics, there is also an inner moment $\mu_{z}$. Since there is no new degree of freedom and the inner moment is proportional to the second derivative of $U_{x}$, the generic frame continuum is a second grade medium. To make this point clearer, the balance equations of a second grade medium (see for instance [12] or [44]) are recalled afterwards in statics with the notation of Germain:

$$
\frac{\partial}{\partial x_{j}}\left(\sigma_{i j}-\frac{\partial \nu_{i j k}}{\partial x_{k}}\right)+f_{i}=0
$$

where $\left(x_{1}, x_{2}\right)=(x, y)$ and the Einstein summation convention over repeated subscripts is used. The vector $\vec{f}$ designates a volumic external force. The symmetric second order tensor $\sigma$ is the intrinsic stress tensor and the third order tensor $\nu$ is the second stress tensor or hyperstress tensor. The comparison with Eq. (32) shows that there exist hyperstresses in the generic frame continuum. But the second grade effect occurs in a single direction and only one component of $\nu$ is different from zero. It is related to the inner moment:

$$
\nu_{x y y}=-\mu_{z}=E I_{z} \frac{\partial^{2} U_{x}}{\partial y^{2}}
$$

This is another illustration of the extreme anisotropy of frame materials. This behavior differs from the one of continuous materials reinforced by linear fibers. 
Even if the inclusions are oriented in a single direction, second grade effects appear in all directions [20]. The second difference with [12] is the presence in the balance equations (32) of frequency dependent functions. The effect of the microstructure is taken into account in the definition of the inertial terms in [12], but it is assumed that every particle has a homogeneous deformation and there is no local resonance.

The generic frame continuum satisfies atypical boundary conditions in addition to the usual boundary conditions. They are similar to the boundary conditions of Euler-Bernoulli beams: bending moment, shear force and gradient of the horizontal displacement $U_{x}$, also see on this point similar situations described in $[31,39]$. The constitutive law is given by Eqs. (30) and (31). As the generic frame continuum is a generalization of SC materials, the elastic moduli in the horizontal and vertical directions can be different. Note that the use of generalized continuum theories requires additional material constants. Their determination is a common difficulty which is overcame by homogenization. Here we obtain directly the analytic expression of the second grade modulus.

When the real values of the parameters are introduced in this model, some terms become negligible and the generic frame continuum degenerates into the previous continua. For example, at low frequencies, $f(\hat{\omega}) \approx 1$ and the inertial terms in Eq. $(m)$ of $(32)$ vanish. The expressions of the node rotation and the shear stresses are then:

$$
\begin{gathered}
\theta=\frac{G_{f}}{G_{w}+G_{f}} \frac{\partial U_{y}}{\partial x}-\frac{G_{w}}{G_{w}+G_{f}} \frac{\partial U_{x}}{\partial y} \\
\tau_{x y}=\tau_{y x}=G\left(\frac{\partial U_{y}}{\partial x}+\frac{\partial U_{x}}{\partial y}\right) \quad \text { with } \quad \frac{1}{G}=\frac{1}{G_{w}}+\frac{1}{G_{f}}
\end{gathered}
$$

If in addition, we assume that the walls and the floors have similar properties, the derivative of the inner moment $\partial_{y} \mu_{z}$ is negligible compared to the shear 
stress $\tau_{x y}$ and we obtain:

$$
\begin{aligned}
& \frac{\partial \sigma_{x x}}{\partial x}+\frac{\partial \tau_{x y}}{\partial y}+\left[M_{w}+M_{f}\right] \omega^{2} U_{x}=0 \\
& \frac{\partial \sigma_{y y}}{\partial y}+\frac{\partial \tau_{y x}}{\partial x}+\left[M_{w}+M_{f}\right] \omega^{2} U_{y}=0
\end{aligned}
$$

This corresponds to the balance equations derived in [17] at low frequencies. The equivalent continuum is a Cauchy medium with an anisotropic constitutive law.

If we now consider a material with the walls much thicker than the floors, the mass of the floors $M_{f}$ becomes negligible and the expressions (35) of the node rotation and the shear stresses degenerate:

$$
G_{f}<<G_{w} \Rightarrow \theta \approx-\frac{\partial U_{x}}{\partial y} \quad \text { and } \quad \tau_{x y}=\tau_{y x} \approx G_{f}\left(\frac{\partial U_{y}}{\partial x}+\frac{\partial U_{x}}{\partial y}\right)
$$

Moreover, for the contrast given by Eqs. (2), the shear stress $\tau_{x y}$ is much smaller than $\partial_{y} \mu_{z}$ and we obtain the balance equations of Sections 3,4 and 5 :

$$
\begin{aligned}
& \frac{\partial \sigma_{x x}}{\partial x}+\frac{\partial^{2} \mu_{z}}{\partial y^{2}}+M_{w} \omega^{2} U_{x}=0 \\
& \frac{\partial \sigma_{y y}}{\partial y}+\frac{\partial \tau_{y x}}{\partial x}+M_{w} \omega^{2} U_{y}=0
\end{aligned}
$$

On the contrary, for the contrast given by Eqs. (2a) and (27) of Section 8.3, the shear stress $\tau_{x y}$ and $\partial_{y} \mu_{z}$ have the same order of magnitude:

$$
\begin{aligned}
& \frac{\partial \sigma_{x x}}{\partial x}+\frac{\partial}{\partial y}\left(\tau_{x y}+\frac{\partial \mu_{z}}{\partial y}\right)+M_{w} \omega^{2} U_{x}=0 \\
& \frac{\partial \sigma_{y y}}{\partial y}+\frac{\partial \tau_{y x}}{\partial x}+M_{w} \omega^{2} U_{y}=0
\end{aligned}
$$

Equations (38) and (39) describe anisotropic second grade media with vanishing terms in the stress tensors.

At higher frequencies, some inertial terms appear in Eq. $(m)$ of (32). As a result, the shear stresses are different and they are equal to quantities which depend on the frequency. It is not possible to separate the elastic effects and the inertial effects. However, the macroscopic behavior of the material is governed 
by the tension-compression of the elements and the influence of the shear is limited. For the normalization used in [17] [see Eqs. (2a) and Tab. 2], the elements are also in resonance in bending and their effective mass has to be taken into account:

$$
\begin{aligned}
& \frac{\partial \sigma_{x x}}{\partial x}+\frac{\partial \hat{\tau}_{x y}(\omega)}{\partial y}+\left[M_{w} f\left(\hat{\omega}_{w}\right)+M_{f}\right] \omega^{2} U_{x}=0 \\
& \frac{\partial \sigma_{y y}}{\partial y}+\frac{\partial \hat{\tau}_{y x}(\omega)}{\partial x}+\left[M_{w}+M_{f} f\left(\hat{\omega}_{f}\right)\right] \omega^{2} U_{y}=0
\end{aligned}
$$

This corresponds to an anisotropic Cauchy medium with an effective mass. The introduction of a contrast between the elements [Eqs. (2)] leads to a second grade medium with an effective mass. In that case, only the floors are in resonance:

$$
\begin{aligned}
& \frac{\partial \sigma_{x x}}{\partial x}+\frac{\partial}{\partial y}\left(\hat{\tau}_{x y}(\omega)+\frac{\partial \mu_{z}}{\partial y}\right)+M_{w} \omega^{2} U_{x}=0 \\
& \frac{\partial \sigma_{y y}}{\partial y}+\frac{\partial \hat{\tau}_{y x}(\omega)}{\partial x}+\left[M_{w}+M_{f} f\left(\hat{\omega}_{f}\right)\right] \omega^{2} U_{y}=0
\end{aligned}
$$

The functions $\hat{\tau}_{x y}(\omega)$ and $\hat{\tau}_{y x}(\omega)$ have not the same expressions as in Eqs. (40) because the balance of moment of momentum is different in the two cases. At most of the frequencies, the effective mass of the floors is negligible in Eq. (y) of (41). It is written to remind that it becomes infinite close to the frequencies of the odd bending modes of the floors.

\section{Conclusion}

This work has emphasized that frame materials have a rich behavior even when the condition of scale separation is respected. It was illustrated by the study of the propagation of plane waves in a material with walls stiffer than floors. In that case, walls can experience bending at the macroscopic scale. The equivalent continuum is a second grade medium at the leading order as for linear fiber reinforced composites $[18,19,20]$. But, contrary to the composites made of continuous materials, the second grade effect only appears in one direction in frame materials. As a result, there is dispersion for the shear waves traveling 
in the direction of the walls and the anisotropy is strongly marked. The shear waves in the direction of the floors are generated by another mechanism called local bending. It is the same mechanism as for the shear waves in materials with similar walls and floors [17]. It consists of the bending of the elements between two neighboring nodes. Since the rigidities of the two types of bending have different orders of magnitude, the shear waves in the direction of the floors appear in another frequency range. Moreover there is no dispersion.

Likewise, shear-compression waves appear in different frequency ranges according to their polarization because they are generated by two mechanisms: the tension-compression of the floors and the tension-compression of the walls. These waves might also be associated with the local resonance in bending of the elements if the frequency ranges coincide. This causes additional kinematic conditions close to the frequencies of the even modes of the resonating elements. Moreover, if these elements are orthogonal to the direction of polarization, the properties of the macroscopic waves depend on an effective mass which is a function of the frequency. Consequently, there are bandgaps close to the frequencies of the odd modes of the resonating elements and dispersion. The latter differs from the dispersion associated with the macroscopic bending of the walls. Its importance is determined by the distribution of mass between the elements.

These results were established thanks to the homogenization method of periodic discrete media (HPDM) coupled with normalization. The homogenization of highly contrasted materials creates mathematical difficulties which are out of the scope of this paper. Since every mechanism has a rigidity with a different order of magnitude, the higher order equations have to be considered to obtain a complete macroscopic description. Thus, the study of the convergence of the solutions is a singular perturbation problem. In addition, the normalization is not valid close to the resonant frequencies of the elements because the order of magnitude of some terms in the higher order equations changes.

Nevertheless, this approach is a powerful tool to observe the various behav- 
iors of a class of reticulated materials. As it is always possible to come back at the local scale to analyze the deformation of the unit cell, the mechanisms associated with each kinematics can be identified. Moreover, the analytic formulation provides a clear understanding of the role of every parameter, not only the thicknesses but also the other characteristics of the elements. However, the HPDM method is not convenient for the direct study of a given material. The main problem is that the scale ratio $\tilde{\epsilon}$, which is necessary to introduce the local physics during the normalization, is estimated at the end of the homogenization process once the equivalent continuum is known. This is the reason why we proposed a dimensionless criterion to compare the possible mechanisms and identify the macroscopic behavior. This criterion depends on the frequency. A given frame material which behaves as a "classical" medium at low frequencies can become a second grade medium at higher frequencies. This provides a framework for the study of real materials or the design of new materials (for example filters) with prescribed properties.

This framework is valid for the four types of waves described in this paper but there probably exist some others. The periodicity of the kinematic descriptors was assumed identical to the geometric periodicity. Other researches on the extension of homogenization to high frequencies $[45,46,47,48]$ suggest that this hypothesis is too restrictive and that the kinematic descriptors are often periodic on two cells or more [49]. The same approach could also be used to investigate the behavior of other classes of reticulated materials. The results would certainly be very similar for three-dimensional frames or materials with a unit cell in the shape of a parallelogram. However, for braced materials such as triangular lattices, the shear mechanisms are probably different. 


\section{A Homogenization method of periodic discrete media}

This appendix describes the implementation of the homogenization method of periodic discrete media in the case of frame materials. A more general presentation of the method can be found in [24] and other examples of the homogenization of frame materials are given in $[29,17]$. The method includes two main steps: the discretization of the dynamic balance followed by the homogenization process.

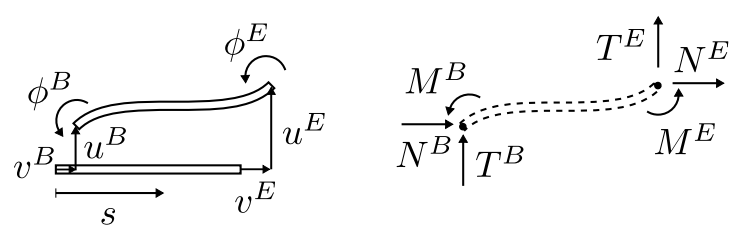

Figure 11: Notation for a frame element

The aim of the first step is to concentrate on the nodes the study of the momentum balance without loss of information. The reticulated material is considered as a periodic lattice of interconnected beams. The element linking the node $B$ to the node $E$ is drawn in figure 11. It is characterized by the parameters $\ell, A$, and $I$. In the local beam frame, $s$ stands for the coordinate along the beam axis, $u, v$ for the transverse and axial displacements respectively, and $\phi$ for the cross-section rotation. The primes denote the differentiation with respect to $s$. The axial force $N$, the shear force $T$, and the bending moment $M$ act by convention from the left part to the right part. No external force is applied on the element which behaves as an Euler-Bernoulli beam. With this notation, the vibrations of the element in harmonic regime are described by the following equations. 
Longitudinal vibrations:

$$
\left\{\begin{array}{c}
N^{\prime}(s)=\rho A \omega^{2} v(s) \\
N(s)=-E A v^{\prime}(s)
\end{array} \quad \Rightarrow \quad \begin{array}{c}
v^{\prime \prime}(s)=-\chi^{2} v(s) \\
\text { where } \chi=\sqrt{\frac{\rho \omega^{2}}{E}}=\frac{2 \pi}{\lambda_{c}}
\end{array}\right.
$$

Transverse vibrations:

$$
\left\{\begin{aligned}
T^{\prime}(s) & =\rho A \omega^{2} u(s) \\
M^{\prime}(s) & =-T(s) \\
M(s) & =-E I u^{\prime \prime}(s)
\end{aligned} \quad \Rightarrow \quad \begin{array}{rl}
u^{\prime \prime \prime \prime}(s)=\beta^{4} u(s) \\
\text { where } \beta=\sqrt[4]{\frac{\rho A \omega^{2}}{E I}}=\frac{2 \pi}{\lambda_{b}}
\end{array}\right.
$$

where $\lambda_{c}$ and $\lambda_{b}$ are the compression wavelength and the bending wavelength, respectively, in the element at the studied circular frequency $\omega$. Both wavelengths are related by a purely geometric relationship:

$$
\lambda_{b}^{2}=\lambda_{c} 2 \pi \sqrt{\frac{I}{A}}=\lambda_{c} \frac{2 \pi a}{\sqrt{12}} \quad \Rightarrow \quad \frac{\lambda_{b}}{\lambda_{c}}=O\left(\frac{a}{\lambda_{b}}\right)<<1
$$

Indeed, the Euler-Bernoulli beam description requires that the bending wavelength is much greater than the thickness of the element. Thus, the bending wavelength is always smaller than the compression wavelength.

Equations (42) and (43) are integrated between the nodes B and E using the unknown displacements and rotations of the endpoints $\left(u^{B}, v^{B}, \phi^{B}\right.$ and $u^{E}$, $\left.v^{E}, \phi^{E}\right)$ as boundary conditions. This provides the expressions of the forces at the extremities of the element in its local frame:

$$
\begin{array}{rlrl}
N^{B} & =N\left(v^{B}, v^{E}\right) & N^{E}=-N\left(v^{E}, v^{B}\right) \\
T^{B}=T\left(u^{B}, u^{E}, \phi^{B}, \phi^{E}\right) & T^{E}=T\left(-u^{E},-u^{B}, \phi^{E}, \phi^{B}\right) \\
M^{B}=M\left(u^{B}, u^{E}, \phi^{B}, \phi^{E}\right) & M^{E}=M\left(u^{E}, u^{B},-\phi^{E},-\phi^{B}\right)
\end{array}
$$


where

$$
\begin{gathered}
N\left(v_{1}, v_{2}\right)=\frac{E A \chi}{\sin (\chi \ell)}\left(v_{1} \cos (\chi \ell)-v_{2}\right) \\
T\left(u_{1}, u_{2}, \phi_{1}, \phi_{2}\right)=\frac{E I \beta^{3}}{1-\cos (\beta \ell) \cosh (\beta \ell)}\left(u_{1}(\cosh (\beta \ell) \sin (\beta \ell)+\sinh (\beta \ell) \cos (\beta \ell))\right. \\
\left.-u_{2}(\sin (\beta \ell)+\sinh (\beta \ell))+\frac{\phi_{1}}{\beta} \sin (\beta \ell) \sinh (\beta \ell)-\frac{\phi_{2}}{\beta}(\cos (\beta \ell)-\cosh (\beta \ell))\right) \\
M\left(u_{1}, u_{2}, \phi_{1}, \phi_{2}\right)=\frac{E I \beta^{2}}{1-\cos (\beta \ell) \cosh (\beta \ell)}\left(u_{1} \sin (\beta \ell) \sinh (\beta \ell)+u_{2}(\cos (\beta \ell)-\cosh (\beta \ell))\right. \\
\left.+\frac{\phi_{1}}{\beta}(\cosh (\beta \ell) \sin (\beta \ell)-\sinh (\beta \ell) \cos (\beta \ell))-\frac{\phi_{2}}{\beta}(\sin (\beta \ell)-\sinh (\beta \ell))\right)
\end{gathered}
$$

The dynamic balance of each element being satisfied, it remains to introduce the geometry of the reticulated material by writing the balance of the nodes. As their mass is negligible and there is no external force, it consists in adding the forces or moments applied by the two walls and the two floors connected to the same node. Moreover, the connection is perfectly stiff and the motions of the four elements are identical. Therefore, the nodal balance equations are a set of finite difference equations expressed in the global frame $(x, y)$ of figure 2 and depending only on the nodal kinematic variables: $U_{x}^{(w, f)}, U_{y}^{(w, f)}$ and $\theta^{(w, f)}$.

Balance of momentum in the $x$-direction:

$$
\begin{aligned}
& T_{w}^{E}\left(U_{x}^{(w, f-1)}, U_{x}^{(w, f)},-\theta^{(w, f-1)},-\theta^{(w, f)}\right) \\
& -T_{w}^{B}\left(U_{x}^{(w, f)}, U_{x}^{(w, f+1)},-\theta^{(w, f)},-\theta^{(w, f+1)}\right) \\
& +N_{f}^{E}\left(U_{x}^{(w-1, f)}, U_{x}^{(w, f)}\right)-N_{f}^{B}\left(U_{x}^{(w, f)}, U_{x}^{(w+1, f)}\right)=0
\end{aligned}
$$

Balance of momentum in the $y$-direction:

$$
\begin{aligned}
& N_{w}^{E}\left(U_{y}^{(w, f-1)}, U_{y}^{(w, f)}\right)-N_{w}^{B}\left(U_{y}^{(w, f)}, U_{y}^{(w, f+1)}\right) \\
& +T_{f}^{E}\left(U_{y}^{(w-1, f)}, U_{y}^{(w, f)}, \theta^{(w-1, f)}, \theta^{(w, f)}\right) \\
& -T_{f}^{B}\left(U_{y}^{(w, f)}, U_{y}^{(w+1, f)}, \theta^{(w, f)}, \theta^{(w+1, f)}\right)=0
\end{aligned}
$$


Balance of moment of momentum:

$$
\begin{aligned}
& M_{w}^{E}\left(-U_{x}^{(w, f-1)},-U_{x}^{(w, f)}, \theta^{(w, f-1)}, \theta^{(w, f)}\right) \\
& -M_{w}^{B}\left(-U_{x}^{(w, f)},-U_{x}^{(w, f+1)}, \theta^{(w, f)}, \theta^{(w, f+1)}\right) \\
& +M_{f}^{E}\left(U_{y}^{(w-1, f)}, U_{y}^{(w, f)}, \theta^{(w-1, f)}, \theta^{(w, f)}\right) \\
& -M_{f}^{B}\left(U_{y}^{(w, f)}, U_{y}^{(w+1, f)}, \theta^{(w, f)}, \theta^{(w+1, f)}\right)=0
\end{aligned}
$$

Once these equations are solved and the nodal kinematic variables are determined, the motions $u, v, \phi$, the forces $N, T$ and the moment $M$ inside each element can always be calculated thanks to Eqs. (42) and (43). The discrete description constituted by Eqs. (47) is a complete description of the material which contains all the necessary information. It is thus possible to use the nodal kinematic variables as descriptors of the motion of the material.

The principles of homogenization are then used to turn the discrete description into a continuous description valid at the macroscopic scale. The aim is to obtain the differential equations describing the behavior of the equivalent continuum. The key assumption is scale separation. This means that the cell size $\ell_{c}$ (here $\ell_{c}=\ell_{w}$ by convention) is small compared to the unknown characteristic size $L$ of the deformation of the frame material under vibrations. Consequently, the scale ratio $\epsilon=\ell_{c} / L$ is a small parameter $(\epsilon<<1)$ used for the expansion of the kinematic descriptors and the forces.

The classical homogenization considers frequencies much smaller than the natural frequencies of the elements of the unit cell. In that case, the condition of scale separation is respected and both wavelengths $\lambda_{c}$ and $\lambda_{b}$ are much longer than $\ell$. It is therefore possible to expand the trigonometric and hyperbolic functions according to the small parameters $\chi \ell$ and $\beta \ell$ in the expressions of the 
nodal forces and moment given in Eq. (46).

$$
\begin{gathered}
N\left(v_{1}, v_{2}\right)=\frac{E A}{\ell}\left(\left(v_{1}-v_{2}\right)-\frac{(\chi \ell)^{2}}{6}\left(2 v_{1}+v_{2}\right)-\frac{(\chi \ell)^{4}}{360}\left(8 v_{1}+7 v_{2}\right)\right)+O\left((\chi \ell)^{6}\right) \\
T\left(u_{1}, u_{2}, \phi_{1}, \phi_{2}\right)=-\frac{12 E I}{\ell^{3}}\left(\left(u_{1}-u_{2}\right)+\frac{\ell}{2}\left(\phi_{1}+\phi_{2}\right)-\frac{(\beta \ell)^{4}}{840}\left(26 u_{1}+9 u_{2}\right)\right. \\
\left.-\frac{(\beta \ell)^{4} \ell}{5040}\left(22 \phi_{1}-13 \phi_{2}\right)\right)+O\left((\beta \ell)^{8}\right) \\
M\left(u_{1}, u_{2}, \phi_{1}, \phi_{2}\right)=\frac{6 E I}{\ell^{2}}\left(\left(u_{1}-u_{2}\right)+\frac{\ell}{3}\left(2 \phi_{1}+\phi_{2}\right)-\frac{(\beta \ell)^{4}}{2520}\left(22 u_{1}+13 u_{2}\right)\right. \\
\left.-\frac{(\beta \ell)^{4} \ell}{2520}\left(4 \phi_{1}-3 \phi_{2}\right)\right)+O\left((\beta \ell)^{8}\right)
\end{gathered}
$$

However, having a quasi-static state at the local scale is only a sufficient condition and homogenization can be extended to situations with local resonance [17]. As $\lambda_{b}$ is always smaller than $\lambda_{c}$ in beams, there is a frequency range with $\lambda_{b}=O(\ell)$ and $\lambda_{c}>>\ell$. The corresponding elements are in resonance in bending and the expansion of the shear force and the bending moment is no longer possible. But we can expand the axial force and apply homogenization. This method is also valid at higher frequencies [with $\lambda_{b}<O(\ell)$ ] provided that $\lambda_{c}$ remains sufficiently long to define a macroscopic scale and that the behavior of the elements is correctly described by the beam model.

Another consequence of scale separation is that the nodal motions slowly vary from one node to the next. The kinematic descriptors can therefore be considered as the discrete values of continuous functions of the space variables $x$ and $y$. These functions are assumed to converge as $\epsilon$ approaches zero and are replaced by asymptotic expansions in powers of $\epsilon$ [Eq. (1)]. Equations (47) describing the balance of a node also depend on the motions of the four neighboring nodes. The distances between the nodes are constant because of the periodicity of the material. They are equal to $\ell_{w}=\epsilon L$ in the vertical direction and to $\ell_{f}=\epsilon \ell^{*} L$ where $\ell^{*}=\ell_{f} / \ell_{w}=O(1)$ in the horizontal direction. These values are small with respect to $x$ and $y$, which enables the variations of the motions to be expressed with Taylor's series. This introduces the macroscopic 
derivatives.

$$
\begin{aligned}
& X^{(w, f \pm 1)}=X^{0}\left(w \ell_{f}, f \ell_{w}\right)+\epsilon\left(X^{1}\left(w \ell_{f}, f \ell_{w}\right) \pm L \frac{\partial X^{0}}{\partial y}\left(w \ell_{f}, f \ell_{w}\right)\right)+\ldots \\
& X^{(w \pm 1, f)}=X^{0}\left(w \ell_{f}, f \ell_{w}\right)+\epsilon\left(X^{1}\left(w \ell_{f}, f \ell_{w}\right) \pm \ell^{*} L \frac{\partial X^{0}}{\partial x}\left(w \ell_{f}, f \ell_{w}\right)\right)+\ldots
\end{aligned}
$$

where $X$ stands for $U_{x}, U_{y}$ or $\theta$.

To correctly take into account the local physics, the physical parameters have to be scaled according to the powers of $\epsilon$. This normalization ensures that each mechanical effect appears at the same order whatever the value of $\epsilon$. Thus, the same physics is kept in the homogenized model, that is to say at the limit when $\epsilon$ is made to approach zero. The choice of the properties of the elements determines the stiffness contrast between the various mechanisms and then the nature of the equivalent continuum. The order of magnitude of the frequency is imposed by the balance of the elastic and inertia forces at the macroscopic level. Since the elastic forces can have several origins, there are several possibilities for the frequency.

Finally, all the expansions in powers of $\epsilon$ and the normalization of the parameters are introduced in the equations describing the balance of the nodes. The obtained relations being valid for any small enough $\epsilon$, the orders can be separated. This leads to a set of differential equations for each order, which can be solved in increasing order. The leading order, which corresponds to the limit when $\epsilon$ approaches zero gives the homogenized model. The superior orders are correctors which improve the accuracy in the case of a poor scale separation.

Funding acknowledgement: This research received no specific grant from any funding agency in the public, commercial, or not-for-profit sectors. 


\section{References}

[1] Z. P. Bažant and M. Christensen. Analogy between micropolar continuum and grid frameworks under initial stress. International Journal of Solids and Structures, 8(3):327-346, 1972.

[2] A. K. Noor. Continuum modeling for repetitive lattice structures. Applied Mechanics Reviews, 41(7):285-296, 1988.

[3] R. S. Kumar and D. L. McDowell. Generalized continuum modeling of 2-D periodic cellular solids. International Journal of Solids and Structures, 41(26):7399-7422, 2004

[4] E. Cosserat and F. Cosserat. Théorie des corps déformables (Theory of deformable bodies). A. Hermann et fils, Paris, 1909.

[5] A. C. Eringen. Linear theory of micropolar elasticity. Journal of Mathematics and Mechanics, 15:909-923, 1966.

[6] A. C. Eringen and E. S. Suhubi. Nonlinear theory of simple micro-elastic solids-I. International Journal of Engineering Science, 2(2):189-203, 1964.

[7] E. S. Suhubi and A. C. Eringen. Nonlinear theory of micro-elastic solids-II. International Journal of Engineering Science, 2(4):389-404, 1964.

[8] R. D. Mindlin. Micro-structure in linear elasticity. Archive for Rational Mechanics and Analysis, 16(1):51-78, 1964.

[9] R.A. Toupin. Elastic materials with couple-stresses. Archive for Rational Mechanics and Analysis, 11:385-414, 1962.

[10] R. D. Mindlin and N. N. Eshel. On first strain-gradient theories in linear elasticity. International Journal of Solids and Structures, 4(1):109 - 124, 1968. 
[11] J-J Alibert, P. Seppecher, and F. dell'Isola. Truss modular beams with deformation energy depending on higher displacement gradients. Mathematics and Mechanics of Solids, 8(1):51-73, 2003.

[12] P. Germain. The method of virtual power in continuum mechanics. Part 2: Microstructure. SIAM Journal on Applied Mathematics, 25(3):556 - 575, 1973.

[13] G. A. Maugin. A historical perspective of generalized continuum mechanics. In Holm Altenbach, Gérard A. Maugin, and Vladimir Erofeev, editors, Mechanics of Generalized Continua, volume 7 of Advanced Structured Materials, pages 3 - 19. Springer Berlin Heidelberg, 2011.

[14] C. Tekoglu and P. R. Onck. Size effects in two-dimensional Voronoi foams: A comparison between generalized continua and discrete models. Journal of the Mechanics and Physics of Solids, 56(12):3541-3564, 2008.

[15] F. Pradel. Homogénéisation des milieux discrets périodiques orientés. Une application aux mousses (Homogenization of discrete periodic oriented media. An applications to foams). PhD thesis, École Nationale des Ponts et Chaussées, 1998.

[16] P. G. Martinsson and I. Babuška. Homogenization of materials with periodic truss or frame micro-structures. Mathematical Models and Methods in Applied Sciences, 17(5):805-832, 2007.

[17] C. Chesnais, C. Boutin, and S. Hans. Effects of the local resonance on the wave propagation in periodic frame structures: Generalized Newtonian mechanics. Journal of the Acoustical Society of America, 132(4, Part 2):2873-2886, 2012.

[18] C. Pideri and P. Seppecher. A second gradient material resulting from the homogenization of an heterogeneous linear elastic medium. Continuum Mechanics and Thermodynamics, 9(5):241-257, 1997. 
[19] B. Sudret and P. de Buhan. A multiphase model for materials reinforced by linear inclusions. Comptes Rendus de l'Académie des Sciences - Series IIB, 327(1):7-12, 1999.

[20] C. Boutin and J. Soubestre. Generalized inner bending continua for linear fiber reinforced materials. International Journal of Solids and Structures, 48(3-4):517-534, 2011.

[21] C. Boutin, J. Soubestre, Dietz M. S., and Taylor C. Experimental evidence of the high-gradient behaviour of fiber reinforced materials. European Journal of Mechanics A/Solids, 42(0):280-298, 2013.

[22] M. Ferretti, Madeo A., F. dell'Isola, and P. Boisse. Modeling the onset of shear boundary layers in fibrous composite reinforcements by secondgradient theory. ZAMP - Journal of Applied Mathematics and Physics, ():303-308, 2013.

[23] D. Caillerie, P. Trompette, and P. Verna. Homogenisation of periodic trusses. In IASS Symposium, 10 Years of Progress in Shell and Spatial Structures, Madrid, 1989.

[24] H. Tollenaere and D. Caillerie. Continuous modeling of lattice structures by homogenization. Advances in Engineering Software, 29(7-9):699-705, 1998.

[25] G. Moreau and D. Caillerie. Continuum modeling of lattice structures in large displacement applications to buckling analysis. Computers and Structures, 68(1-3):181-189, 1998.

[26] D. Caillerie, A. Mourad, and A. Raoult. Cell-to-muscle homogenization. Application to a constitutive law for the myocardium. ESAIMMathematical Modelling and Numerical Analysis, 37(4):681-698, 2003.

[27] D. Caillerie, A. Mourad, and A. Raoult. Discrete homogenization in graphene sheet modeling. Journal of Elasticity, 84(1):33-68, 2006. 
[28] C. Boutin and S. Hans. Homogenisation of periodic discrete medium: Application to dynamics of framed structures. Computers and Geotechnics, 30(4):303-320, 2003.

[29] S. Hans and C. Boutin. Dynamics of discrete framed structures: A unified homogenized description. Journal of Mechanics of Materials and Structures, 3(9):1709-1739, 112008.

[30] F. dell'Isola and P. Seppecher. The relationship between edge contact forces, double forces and interstitial working allowed by the principle of virtual power. Comptes Rendus de l'Académie des Sciences - Series IIB, 321(8):1-26, 1995.

[31] F. dell'Isola, A. Madeo, and L. Placidi. Linear plane wave propagation and normal transmission and reflection at discontinuity surfaces in second gradient 3D continua. ZAMM - Journal of Applied Mathematics and Mechanics, 92(1):52-71, 2012.

[32] L. Placidi, G. Rosi, I. Giorgio, and A. Madeo. Reflection and transmission of plane waves at surfaces carrying material properties and embedded in second-gradient materials. Mathematics and Mechanics of Solids, ():1-24, 2013.

[33] A. Bensoussan, J-L Lions, and G. Papanicolaou. Asymptotic analysis for periodic structures, volume 5 of Studies in mathematics and its applications. North Holland, Amsterdam, 1978.

[34] E. Sanchez-Palencia. Non-homogeneous media and vibration theory, volume 127 of Lecture notes in physics. Springer-Verlag, Berlin, 1980.

[35] R. Chiheb, D. Cioranescu, A. El Janati, and G. Panasenko. Reinforced reticulated structures in elasticity. Comptes Rendus de l'Académie des Sciences - Series I - Mathematics, 326(7):897-902, 1998. 
[36] D. Cioranescu and J. Saint Jean Paulin. Homogenization of Reticulated Structures, volume 136 of Applied Mathematical Sciences. Springer-Verlag, New York, 1999.

[37] D. Caillerie. General study of a kind of singular stiff problems. Comptes Rendus de l'Académie des Sciences - Series I - Mathematics, 323(7):835840, 1996.

[38] D. Caillerie and E. Sanchez-Palencia. Elastic thin shells - Asymptotic theory in the anisotropic and heterogeneous cases. Mathematical Models and Methods in Applied Sciences, 5(4):473-496, 1995.

[39] J. Soubestre and C. Boutin. Non-local dynamic behavior of linear fiber reinforced materials. Mechanics of Materials, 55(0):16-32, 2012.

[40] J-L Auriault and C. Boutin. Long wavelength inner-resonance cut-off frequencies in elastic composite materials. International Journal of Solids and Structures, 49(23-24):3269-3281, 2012.

[41] C. Boutin and J-L Auriault. Rayleigh scattering in elastic composite materials. International Journal of Engineering Science, 31(12):1669-1689, DEC 1993.

[42] A. S. Phani, J. Woodhouse, and N. A. Fleck. Wave propagation in twodimensional periodic lattices. Journal of the Acoustical Society of America, 119(4):1995-2005, 2006.

[43] C. Boutin and J-L Auriault. Dynamic behaviour of porous media saturated by a viscoelastic fluid. Application to bituminous concretes. International Journal of Engineering Science, 28(11):1157-1181, 1990.

[44] F. dell'Isola, G. Sciarra, and S. Vidoli. Generalized Hookes law for isotropic second gradient materials. Proceedings of the Royal Society A, 465(2107):2177-2196, 2009. 
[45] N. Moustaghfir, E. M. Daya, B. Braikat, N. Damil, and M. Potier-Ferry. Evaluation of continuous modelings for the modulated vibration modes of long repetitive structures. International Journal of Solids and Structures, 44(21):7061-7072, 2007.

[46] R. V. Craster, J. Kaplunov, and A. V. Pichugin. High-frequency homogenization for periodic media. Proceedings of the Royal Society A, 466(2120):2341-2362, 2010.

[47] R. V. Craster, J. Kaplunov, and J. Postnova. High-frequency asymptotics, homogenisation and localisation for lattices. Quarterly Journal of Mechanics and Applied Mathematics, 63(4):497-519, 2010.

[48] I. L. Manevitch and G. V. Oshmyan. An asymptotic study of the linear vibrations of a stretched beam with concentrated masses and discrete elastic supports. Journal of Sound and Vibration, 223(5):679 - 691, 1999.

[49] C. Boutin, A. Rallu, and S. Hans. Large scale modulation of high frequency acoustic waves in periodic porous media. Journal of the Acoustical Society of America, 132(6):3622-3636, 2012.

\section{List of Figures}

1 Examples of frame materials . . . . . . . . . . . . . . 2

2 Notation for the frame material . . . . . . . . . . . . 5

3 Comparison of the two types of bending . . . . . . . . . . . 11

4 Shear wave generated by the local bending of the floors . . . . . 14

5 Shear wave generated by the propagation of bending waves in the walls 16

$6 \quad$ Variation of the wave number for shear waves in the $y$ direction . 17

$7 \quad$ Macroscopic shear-compression wave traveling in the direction $\vec{n}_{\alpha}$ with the X-mode 19

8 Variations of the function $f$ according to the dimensionless frequency 22

9 Macroscopic shear-compression wave traveling in the direction $\vec{n}_{\alpha}$ with the Y-mode 26 
10 Variation of the wave number for the Y-mode and $M_{f}=M_{w} / 10 \quad 29$

11 Notation for a frame element . . . . . . . . . . . . . 48 